\title{
STRUCTURE OF THE TENSOR PRODUCT SEMIGROUP*
}

\author{
MISHA KAPOVICH ${ }^{\dagger}$ AND JOHN J. MILLSON ${ }^{\ddagger}$
}

To the memory of $S$. S. Chern

\begin{abstract}
We study the structure of the semigroup Tens $(G)$ consisting of triples of dominant weights $(\lambda, \mu, \nu)$ of a complex reductive Lie group $G$ such that

$$
\left(V_{\lambda} \otimes V_{\mu} \otimes V_{\nu}\right)^{G} \neq 0
$$

We prove two general structural results for $\operatorname{Tens}(G)$ and compute $\operatorname{Tens}(G)$ for $G=S p(4, \mathbb{C})$ and $G=G_{2}$.
\end{abstract}

Key words. tensor products; irreducible representations

AMS subject classifications. 22E46, 20E42, 17B10

1. Introduction. Suppose that $G$ is a complex reductive Lie group. Finitedimensional irreducible representations $V_{\lambda}$ of $G$ are parameterized by their highest weights $\lambda \in \Delta \cap L$, where $\Delta$ is the positive Weyl chamber and $L$ is the character lattice of a maximal (split) torus in $G$. One of the basic questions of the representation theory is to decompose tensor products $V_{\lambda} \otimes V_{\mu}$ into sums of irreducible representations. Accordingly, we define the set

$$
\operatorname{Tens}(G):=\left\{(\lambda, \mu, \nu) \in(\Delta \cap L)^{3}:\left(V_{\lambda} \otimes V_{\mu} \otimes V_{\nu}\right)^{G} \neq 0\right\} .
$$

For a simply-connected Lie group $G$ with root system $R$ we will write Tens $(R)$ instead of $\operatorname{Tens}(G)$. It has been known for a long time, see for example [12, Theorem 9.8], that the set Tens $(G)$ forms a semigroup with respect to addition. The goal of this paper is to provide more specific structural theorems for Tens $(G)$ and to compute $\operatorname{Tens}(\operatorname{Sp}(4, \mathbb{C}))$ and $\operatorname{Tens}\left(G_{2}\right)$.

ThEOREM 1.1. For each complex reductive Lie group $G$, the set Tens $(G)$ is a finite union of elementary subsets of $L^{3}$.

Here an elementary subset is a subset given by a finite system of linear inequalities (with integer coefficients) and congruences. Thus, to "describe" Tens $(G)$ one would have to find these inequalities and congruences. The above theorem is an analogue of a theorem of C. Laskowski [17], who proved a similar statement for the structure constants of spherical Hecke rings.

Our next theorem provides a glimpse of what these inequalities and congruences might look like. In [2] and [10] the authors defined a finite-sided homogeneous polyhedral cone $\mathcal{P}(G)=D_{3}(G / K) \subset \Delta^{3}$ (where $K$ is a maximal compact subgroup of $G)$, given by the inequalities of the form:

$$
\left\langle\varpi_{i}, w_{1} \lambda\right\rangle+\left\langle\varpi_{i}, w_{2} \mu\right\rangle+\left\langle\varpi_{i}, w_{3} \nu\right\rangle \leq 0
$$

\footnotetext{
*Received July 15, 2005; accepted for publication February 14, 2006.

${ }^{\dagger}$ Department of Mathematics, University of California, Davis, CA 95616, USA (kapovich@ math.ucdavis.edu).

${ }_{\ddagger}$ Department of Mathematics, University of Maryland, College Park, MD 20742, USA (jjm@math.umd.edu).
} 
where $\varpi_{i}$ are the fundamental weights of $R$ and $w_{i}$ are certain elements of the Weyl group of $G$ determined by the "Schubert calculus". It is known (see for instance [12]) that

$$
\operatorname{Tens}(G) \subset \mathcal{P}(G) \cap\left\{\sigma=(\lambda, \mu, \nu) \in L^{3}: \operatorname{Tr}(\sigma) \in Q(R)\right\} .
$$

Here and in what follows $Q(R)$ is the root lattice and $\operatorname{Tr}(\sigma):=\lambda+\mu+\nu$.

THEOREM 1.2. There exists a vector $\sigma \in \mathcal{P}(G)$ such that

$$
(\mathcal{P}(G)+\sigma) \cap \operatorname{Tens}(G)=(\mathcal{P}(G)+\sigma) \cap\left\{(\lambda, \mu, \nu) \in L^{3}: \operatorname{Tr}(\sigma) \in Q(R)\right\} .
$$

In other words, inside the translated cone $\mathcal{P}(G)+\sigma$ the necessary conditions on $(\lambda, \mu, \nu)$ to belong to Tens $(G)$ are also sufficient. Observe that the intersection $\left\{\sigma \in L^{3}: \operatorname{Tr}(\sigma) \in Q(R)\right\} \cap(\mathcal{P}(G)+\sigma)$ is an elementary subset of the lattice $L^{3}$. The length of the vector $\sigma$ can be explicitly estimated. In section 4 we make such an estimate in the case when $R=B_{\ell}$; the same methods work for other root systems.

In section 5 we will compute the semigroup Tens $=\operatorname{Tens}(G)$ for the group $G=S p(4, \mathbb{C})$ and show that this set is not an elementary set itself. Below $P(R)=L$ is the weight lattice of $G$ and $\varpi_{2}$ is the longest fundamental weight. We let

$$
\Lambda:=\left\{\sigma \in L^{3}: \operatorname{Tr}(\sigma) \in Q(R)\right\} .
$$

Then

THEOREM 1.3. $\sigma=(\lambda, \mu, \nu) \in \mathcal{P}(G) \cap \Lambda$ belongs to Tens if and only if one of the following is satisfied:

1. At least two of the vectors $\lambda, \mu, \nu$ are not multiples of $\varpi_{2}$.

2. If two of the vectors $\lambda, \mu, \nu$ are multiples of $\varpi_{2}$ then $\operatorname{Tr}(\sigma) \in 2 P(R)$.

In section 5.4 we express Tens as a union of 6 elementary subsets. We refer the reader to $[10,16]$ (see also section 2.3 of this paper) for the explicit computation of the polyhedra $\mathcal{P}(G)$ for all complex simple Lie group of rank $\leq 3$.

In section 6 we compute the semigroup Tens $(R)$ for the root system $R=G_{2}$.

We note that prior to the present paper, the complete description of the semigroup Tens $(G)$ was known only for the groups of type $A_{n}$. In this case the Saturation Theorem of Knutson and Tao [15] implies that

$$
\operatorname{Tens}(G)=\mathcal{P}(G) \cap\left\{\sigma \in L^{3}: \operatorname{Tr}(\sigma) \in Q(R)\right\} .
$$

Below is a conjecture on the structure of the semigroup Tens $(G)$ for an arbitrary root system $R$. Here $L$ denotes the character lattice of a maximal torus. A triple of dominant weights $\sigma=\left(\lambda_{1}, \lambda_{2}, \lambda_{3}\right)$ is called singular if at least one of the vectors $\lambda_{i}$ is singular, i.e. belongs to a wall of the Weyl chamber $\Delta$.

Conjecture 1.4. 1. Suppose that $R$ is simply-laced. Then

$$
\operatorname{Tens}(G)=\left\{\sigma=\left(\lambda_{1}, \lambda_{2}, \lambda_{3}\right) \in \mathcal{P}(G): \lambda_{i} \in L, \operatorname{Tr}(\sigma) \in Q(R)\right\} .
$$

2. Suppose that $\sigma=\left(\lambda_{1}, \lambda_{2}, \lambda_{3}\right) \in \mathcal{P}(G) \cap L^{3}$ is a nonsingular triple. Then $\sigma \in \operatorname{Tens}(G)$ if and only if $\sigma \in \mathcal{P}(G)$ and $\operatorname{Tr}(\sigma) \in Q(R)$. 
3. Suppose that $P(R)=Q(R)$. Then in the decomposition of Tens $(G)$ as the union of elementary sets, the elementary sets are given by inequalities only and there are no congruence conditions.

The above conjecture holds for the root systems $A_{n}, B_{2}=C_{2}$ and $G_{2}$, it is also supported by a number of computer-based calculations with the higher rank root systems.

REMARK 1.5. Let $T^{\text {reg }}$ be the set of nonsingular triples $\sigma \in \mathcal{P}(G) \cap L^{3}$. It was observed by J. Bernstein that in the decomposition of $T^{\text {reg }}$ into elementary sets, there are no congruence conditions apart from the "obvious" condition $\operatorname{Tr}(\sigma) \in Q(R)$.

A less ambitious conjecture (which actually follows from either Part 1 or 2 of Conjecture 1.4) is

Conjecture 1.6 (S. Kumar). There exists a triple $\sigma \in \mathcal{P}(G) \cap L^{3}, \operatorname{Tr}(\sigma) \in Q(R)$ such that $\sigma \notin$ Tens $(G)$ if and only if there exists a singular triple $\sigma$ with the above properties.

Acknowledgements. During the work on this paper the first author was supported by the NSF grant DMS-04-05180; part of this work was done when he was visiting the Max Plank Institute for Mathematics in Bonn. The second author was supported by the NSF grant DMS-04-05606. The authors gratefully acknowledge support of these institutions.

The possibility of Theorem 1.2 was suggested by J. Bernstein in a conversation at Oberwolfach. T. Haines and S. Kumar told us about Theorem 4.6. The authors are grateful to J. Bernstein, T. Haines, S. Kumar and C. Laskowski for these and other useful conversations. The second author would like to acknowledge how exciting it was to learn Chern-Weil theory and the theory of Chern-Simons invariants first-hand when he was a graduate student in Berkeley in the early seventies.

\section{Review of the path model for the representation theory of complex reductive Lie groups.}

2.1. Root systems and Coxeter complexes. Let $V$ be a finite-dimensional Euclidean vector space and $R \subset V$ be a root system in $V$. Then the collection of coroots $R^{\vee}$ determines a root system in $V^{*}$. Using the metric on $V$ we identify $R^{\vee}$ with a root system in $V$. We will also use the metric to identify elements of $R$ with linear functionals on $V$. Given $R$ we define the affine Coxeter group $W_{\text {aff }}:=W_{R^{\vee}, \text { aff }}$ as the group generated by reflections in the walls

$$
H=\{x \in V: \alpha(x)=t\}, \alpha \in R^{\vee}, t \in \mathbb{Z} .
$$

We let $W=W_{R^{\vee}, s p h}=W_{R, s p h}$ denote the linear part of $W_{R, a f f}$, which is the same as the stabilizer of the origin in $W_{R^{\vee}, a f f}$. The group $W$ is the Weyl group of the root system $R$ (and $R^{\vee}$ ).

REMARK 2.1. In the context of the representation theory of a complex semisimple Lie group $G$, the space $V$ equals $X^{*}(T) \otimes \mathbb{R}$, where $T$ is a maximal torus in $G$. Thus $R \subset V$ and the walls in $V$ are defined by the coroot system $R^{\vee}$ as above.

A Weyl chamber $\Delta$ is a fundamental domain for the finite reflection group $W$, it is bounded by certain walls passing through the origin. Given a vector $v \in \Delta$ we let $v^{*} \in \Delta$ denote the contragredient vector $v^{*}=w_{0}(-v)$, where $w_{0} \in W$ is the longest element, i.e. the element which carries $-\Delta$ to $\Delta$. 
The group $W_{\text {aff }}$ acts by isometries on the Euclidean space $V$. Let $A$ denote the affine space underlying $V$. The pair $\left(A, W_{a f f}\right)$ is called a Euclidean Coxeter complex. Let $\mathcal{W}$ denote the union of all walls. Then the closures of connected components of $A \backslash \mathcal{W}$ are called alcoves. If $R$ is irreducible and spans $V$ then alcoves are simplices and therefore $\left(A, W_{a f f}\right)$ has natural structure of a simplicial complex.

Each alcove is a fundamental domain for the action $W_{\text {aff }} \curvearrowright A$. Pick an alcove $a$ and call it a fundamental alcove. We have a natural projection $\theta: A \rightarrow a$ sending each point $v \in A$ to the unique intersection point $\theta(v) \in W_{a f f} \cdot v \cap a$. The image $\theta(v)$ is called the type of $v$.

Special vertices of the complex $\left(A, W_{a f f}\right)$ are vertices whose stabilizer in $W_{a f f}$ is isomorphic to $W_{s p h}$. The weight group $P(R)$ acts simply transitively on the set of special vertices.

Let $h: A \rightarrow A$ be a dilation of $A$, i.e. an affine map of the form $x \mapsto k x+b$, where $b \in V, k>0$. The number $k$ is the conformal factor of $h$. We define $\operatorname{Dil}\left(A, W_{a f f}\right)$ to be the semigroup of dilations $h$ of $A$ such that

$$
h W_{a f f} h^{-1} \subset W_{a f f} .
$$

Then each $h \in \operatorname{Dil}\left(A, W_{a f f}\right)$ sends vertices of $\left(A, W_{a f f}\right)$ to vertices of $\left(A, W_{a f f}\right)$, walls to walls, etc.

2.2. Chains. Let $R \subset V$ be a root system in a Euclidean vector space $V, W$ be the Weyl group of $R$. We pick a Weyl chamber $\Delta$ for $W$, this determines the set of positive roots $R_{+}$and the set of the simple roots $\Phi$ in $R$, as well as positive and simple coroots. The following notion of chains and the partial order $\geq$ on $V \backslash\{0\}$ was introduced by P. Littelmann in [18].

Definition 2.2. A $W$-chain in $V$ is a finite sequence $\left(\eta_{0}, \ldots, \eta_{m}\right)$ of nonzero vectors in $V$ so that for each $i=1, \ldots, m$ there exists a positive coroot $\beta_{i} \in R^{\vee}$ so that the corresponding reflection $\tau_{i}:=\tau_{\beta_{i}} \in W$ satisfies

1. $\tau_{i}\left(\eta_{i-1}\right)=\eta_{i}$.

2. $\beta_{i}\left(\eta_{i-1}\right)<0$.

Then $\eta \geq_{W} \nu$ if there exists a chain from $\eta$ to $\nu$. Most of the time we will abbreviate $\geq_{W}$ to $\geq$. We say that a chain

$$
\eta_{0}^{\prime} \geq \ldots \eta_{i}^{\prime} \geq \ldots \geq \eta_{n}^{\prime}
$$

is a refinement of the chain

$$
\eta_{0} \geq \ldots \geq \eta_{i} \geq \ldots \geq \eta_{k}
$$

if $\eta_{0}=\eta_{0}^{\prime}, \eta_{n}^{\prime}=\eta_{k}$ and

$$
\left\{\eta_{0}, \ldots, \eta_{k}\right\} \subset\left\{\eta_{0}^{\prime}, \ldots, \eta_{n}^{\prime}\right\}
$$

A chain which does not admit a proper refinement is called maximal.

Define a (nontransitive) relation $\sim$ on $V \backslash\{0\}$ by

$$
\mu \sim_{W} \nu \Longleftrightarrow
$$

$\mu, \nu$ belong to the same Weyl chamber of $W$.

We will write $\sim_{W}$ instead of $\sim$ if there is a possibility of confusion. 
We write $\lambda \gtrsim \xi$ if there exist $\mu, \nu$ so that

$$
\lambda \geq \mu \sim \nu \geq \xi
$$

Accordingly, we define generalized chains as sequences of nonzero vectors in $V$ :

$$
\eta_{0} \gtrsim \eta_{1} \gtrsim \ldots \gtrsim \eta_{m}
$$

2.3. Buildings. Our discussion of buildings follows [14]. We refer the reader to [5], [19], [20] for the more combinatorial discussion.

Fix a spherical or Euclidean (discrete) Coxeter complex $(A, W)$, where $A$ is a Euclidean space $E$ or a unit sphere $S$ and $W=W_{a f f}$ or $W=W_{s p h}$ is a discrete Euclidean or a spherical Coxeter group acting on $A$.

A metric space $Z$ is called geodesic if every pair of points $x, y$ in $Z$ can be connected by a geodesic segment $\overline{x y}$.

Let $Z$ be a metric space. A geometric structure on $Z$ modeled on $(A, W)$ consists of an atlas of isometric embeddings $\varphi: A \hookrightarrow Z$ satisfying the following compatibility condition: For any two charts $\varphi_{1}$ and $\varphi_{2}$, the transition map $\varphi_{2}^{-1} \circ \varphi_{1}$ is the restriction of an isometry in $W$. The charts and their images, $\varphi(A)=a \subset Z$, are called apartments. We will sometimes refer to $A$ as the model apartment. We will require that there are plenty of apartments in the sense that any two points in $Z$ lie in a common apartment. All $W$-invariant notions introduced for the Coxeter complex $(A, W)$, such as rank, walls, singular subspaces, chambers etc., carry over to geometries modeled on $(A, W)$. If $a, a^{\prime} \subset X$ are alcoves (in the Euclidean case) or chambers (in the spherical case) then there exists an apartment $A^{\prime} \subset X$ containing $a \cup a^{\prime}$ : Just take regular points $x \in a, x^{\prime} \in a^{\prime}$ and an apartment $A^{\prime}$ passing through $x$ and $x^{\prime}$.

A geodesic metric space $Z$ is said to be a $C A T(0)$-space (resp, $C A T(1)$-space) if geodesic triangles in $Z$ are "thinner" than geodesic triangles in $\mathbb{R}^{2}$ (resp. in the unit sphere $S^{2}$ ). We refer the reader to [1] for the precise definition.

Definition 2.3. A Euclidean (resp. spherical) building is a CAT(0)-space (resp. CAT(1)-space) modeled on a Euclidean (resp. spherical) Coxeter complex.

A building is called thick if every wall is an intersection of apartments. A nonthick building can always be equipped with a natural structure of a thick building by reducing the Coxeter group.

Let $\mathbb{K}$ be a local field with a (discrete) valuation $\nu$ and valuation ring $\mathcal{O}$. Given a split reductive algebraic group $\underline{G}$ over $\mathbb{Z}$, we obtain the associated group of $\mathbb{K}$-points $G=\underline{G}(\mathbb{K})$. We will call such a group a nonarchimedean Lie group. We can associate with $G$ a Euclidean building (a Bruhat-Tits building) $X=X_{G}$. We refer the reader to [6], [12] and [20] for more detailed discussion of the properties of $X$. Here we only recall that:

1. $X$ is thick and locally compact.

2. $X$ is modeled on a Euclidean Coxeter complex $\left(A, W_{a f f}\right)$ whose dimension equals the rank of $\underline{G}$, and the root system is isomorphic to the root system of $\underline{G}$.

3. $X$ contains a special vertex $o$ whose stabilizer in $G$ is $\underline{G}(\mathcal{O})$.

EXAMPLE 2.4. Let $X$ be a (discrete) Euclidean building, consider the spaces of directions $\Sigma_{x} X$. We will think of this space as the space of germs of non-constant geodesic segments $\overline{x y} \subset X$. As a polysimplicial complex $\Sigma_{x} X$ is just the link of the point $x \in X$. The space of directions has the structure of a spherical building modeled 
on $\left(S, W_{\text {sph }}\right)$, which is thick if and only if $x$ is a special vertex of $X$, see [14]. The same applies in the case when $X$ is a spherical building.

Let $\left(A, W_{a f f}\right)$ be a Euclidean Coxeter complex and pick a Weyl chamber $\Delta \subset A$.

Given a pair of points $x, y \in A$ we define their $\Delta$-distance $d_{\Delta}(x, y)$ by first taking the vector $v:=y-x \in V$. Here we think of $A$ as a principal affine space with underlying vector space $V$ (to be very concrete think of $v$ as a vector with its tail at the origin). We then apply an element $w \in W_{s p h}$ to $v$ chosen so that $u:=w(v) \in \Delta$. Then $d_{\Delta}(x, y):=u$.

Suppose that $X$ is a Euclidean building modeled on $\left(A, W_{\text {aff }}\right)$. We define a $\Delta$ distance in $X$ as follows. For a pair of points $x, y \in X$ pick an apartment $\phi: A \rightarrow$ $A^{\prime} \subset X$ such that $A^{\prime}$ contains $x, y$. Then set

$$
d_{\Delta}(x, y):=d_{\Delta}\left(\phi^{-1}(x), \phi^{-1}(y)\right) .
$$

It is easy to see that this distance is independent of the choice of $\phi$. A similar definition applies if $X=G / K$ is a nonpositively curved symmetric space, where $A$ is a maximal flat in $X$ and $W_{\text {sph }} \curvearrowright A$ is the Weyl group of $G$.

Generalized triangle inequalities. Suppose that $X$ is a nonpositively curved symmetric space or a Euclidean building as above. Define the set

$$
\begin{gathered}
D_{3}(X):=\left\{(\lambda, \mu, \nu) \in \Delta^{3}: \exists \text { a geodesic triangle }[x, y, z] \subset X\right. \text { with } \\
\left.d_{\Delta}(x, y)=\lambda, d_{\Delta}(y, z)=\mu, d_{\Delta}(z, x)=\nu\right\} .
\end{gathered}
$$

It is proven in $[10,11]$ that $D_{3}(X)$ is a convex homogeneous polyhedral cone which depends only on the pair $\left(A, W_{s p h}\right)$, therefore we will frequently use the notation $\mathcal{P}(G)$ for $D_{3}(G / K)$, where $G$ is a reductive Lie group with a maximal compact subgroup $K$. In the case when $G$ is a complex semisimple Lie group, the inequalities defining this polyhedron have the form

1. Stability inequalities $\psi_{j}(\lambda, \mu, \nu) \geq 0$ :

$$
-\left\langle\varpi_{i}, w_{1} \lambda\right\rangle-\left\langle\varpi_{i}, w_{2} \mu\right\rangle-\left\langle\varpi_{i}, w_{3} \nu\right\rangle \geq 0,
$$

where $\varpi_{i}$ are the fundamental weights of $R$ and $w_{i}$ are certain elements of the Weyl group of $G$.

2. Chamber inequalities $\psi_{k}(\lambda, \mu, \nu) \geq 0$ :

$$
\alpha^{\vee}(\lambda) \geq 0, \alpha^{\vee}(\mu) \geq 0, \alpha^{\vee}(\nu) \geq 0
$$

where $\alpha$ are simple roots in $R$.

In [10] the polyhedra $D_{3}(G)$ were computed for all complex semisimple Lie groups $G$ of rank 2. Below we provide the explicit set of stability inequalities for this polyhedron in the case $G=S p(4, \mathbb{C})$.

The Weyl chamber $\Delta$ is given by

$$
\Delta=\{(x, y): x \geq y \geq 0\} .
$$

We will omit these inequalities from the list of inequalities defining $\mathcal{P}(G)$ and will list only the stability inequalities. Instead of the notation $(\lambda, \mu, \nu)$ for elements of $\Delta^{3}$, we will use the more symmetric notation $\left(\lambda_{1}, \lambda_{2}, \lambda_{3}\right)$, where $\lambda_{i}=\left(x_{i}, y_{i}\right), i=1,2,3$. 
The system of stability inequalities defining $\mathcal{P}(S p(4, \mathbb{C}))$ breaks into two subsystems (since $S p(4, \mathbb{C}$ ) has rank 2). The first subsystem is given by

$$
\begin{aligned}
& x_{i} \leq x_{j}+x_{k}, \quad\{i, j, k\}=\{1,2,3\} \\
& y_{i} \leq y_{j}+x_{k}, \quad\{i, j, k\}=\{1,2,3\} .
\end{aligned}
$$

In order to describe the second subsystem we set

$$
S=x_{1}+y_{1}+x_{2}+y_{2}+x_{3}+y_{3}
$$

The second subsystem is then given by

$$
x_{i}+y_{j} \leq S / 2, \quad 1 \leq i, j \leq 3 .
$$

2.4. LS paths and their generalizations. Suppose that $\left(A, W_{a f f}\right)$ is a Euclidean Coxeter complex. Given a point $x \in A$ let $W_{x}$ denote the stabilizer of $x$ in $W_{\text {aff }}$. For a vector $v$ in $V$ define the path $\pi_{v}$ by the formula:

$$
\pi_{v}(t)=t v, \quad t \in[0,1] .
$$

In what follows we will assume that all paths are (re)parameterized to have constant speed and domain $I:=[0,1]$.

Given two paths $p_{1}, p_{2}$ in $A$, we define their concatenation $p=p_{1} * p_{2}$ by

$$
p(t)=\left\{\begin{array}{c}
p_{1}(t), \quad t \in[0,1], \\
p_{1}(1)-p_{2}(0)+p_{2}(t), \quad t \in[1,2] .
\end{array}\right.
$$

Suppose that $p: I \rightarrow A$ is a path and $J=[a, b]$ is nondegenerate subinterval in $I=[0,1]$. We will use the notation $\left.p\right|_{J}$ to denote the restriction of $p$ to $[a, b]$

If $p$ is a PL path in $A$ which is the concatenation

$$
\overline{x_{1} x_{2}} * \ldots * \overline{x_{n} x_{n+1}}
$$

of geodesic segments, then the $\Delta$-length of $p$, denoted length $\Delta(p)$, is defined as the sum

$$
\sum_{i=1}^{n} d_{\Delta}\left(x_{i}, x_{i+1}\right)
$$

Given a PL path $p$ in $A$ we use the notation $p_{ \pm}^{\prime}(t) \in T_{p(t)}(A)$ to denote the derivatives of $p$ at $t$ from the left and from the right.

We say that a path $p:[0,1] \rightarrow A$ is a billiard path if for each $t \in[0,1]$,

$$
p_{-}^{\prime}(t) \in W_{p(t)} p_{+}^{\prime}(t) .
$$

If $p:=p_{1} * \ldots * p_{m}$ is a concatenation of billiard paths then we set

$$
\underline{\text { length }}_{\Delta}(p):=\left(\operatorname{length}_{\Delta}\left(p_{1}\right), \ldots, \text { length }_{\Delta}\left(p_{m}\right)\right) .
$$

Definition 2.5. A PL path $p:[0,1] \rightarrow A$ in $A$ is said to be an $L S$ path with respect to the root system $R$ if:

1. $p(0), p(1) \in P(R)$. 
2. For each $t \in[0,1]$ we have

$$
p_{-}^{\prime}(t) \geq_{W_{p(t)}} p_{+}^{\prime}(t) .
$$

3. There is a $W_{p(t)}$-chain from $p_{-}^{\prime}(t)$ to $p_{+}^{\prime}(t)$ which is maximal as a $W$-chain when we regard $p_{-}^{\prime}(t)$ and $p_{+}^{\prime}(t)$ as vectors in $V$.

REMARK 2.6. This definition is a slight generalization of the Littelmann's definition in [18], where it is assumed that $p(0)=0$.

Definition 2.7. A PL path $p:[0,1] \rightarrow A$ is said to be $a$ Hecke path if it satisfies properties 1 and 2 in Definition 2.5.

Note that each Hecke path is necessarily a billiard path. Let $t_{i}, i=1, \ldots, n$, denote the break-points of $p$, i.e. such that $p$ is not geodesic at $t$. We then obtain a chain

$$
p^{\prime}(0) \geq p_{+}^{\prime}\left(t_{1}\right) \geq \ldots \geq p_{+}^{\prime}\left(t_{n-1}\right) \geq p^{\prime}(1) .
$$

Using property (3) we extend this chain to a maximal $W$-chain

$$
\eta_{0}=p^{\prime}(0) \geq \ldots \geq \eta_{m}=p^{\prime}(1) .
$$

Then the translated path $p-p(0)$ is the concatenation of the geodesic paths

$$
\pi_{a_{i} \eta_{i}}, a_{i} \geq 0, i=1, \ldots, n
$$

We also need a generalization of the concept of an LS path described below:

Definition 2.8 ([13]). Suppose that $p_{1}, \ldots, p_{m}:[0,1] \rightarrow A$ are LS paths. Their concatenation $p_{1} * \ldots * p_{m}$ is called a generalized $L S$ path if for each $i=1, \ldots, m-1$ we have:

$$
\left(p_{i}^{\prime}\right)_{-}(1) \gtrsim\left(p_{i}^{\prime}\right)_{+}(0)
$$

We will use the notation $L S(R)$ and $L S_{1}(R)$ to denote the sets of LS paths and generalized LS paths with respect to the root system $R$. In fact, in this paper we will be using only (generalized) LS paths $p=p_{1} * \ldots * p_{m}$ such that

$$
\underline{\text { length }}_{\Delta}(p)=\left(n_{1} \varpi_{1}, \ldots, n_{m} \varpi_{m}\right), n_{i} \in \mathbb{Z}_{+},
$$

where $\varpi_{i}$ are fundamental weights of $R$.

2.5. The path model. The following theorem is a version of Littelmann's rule for decomposing tensor products:

Theorem 2.9 ([13], Corollary 5.22). The tensor product $V_{\lambda} \otimes V_{\mu}$ contains $V_{\nu}$ as a subrepresentation if and only if there exists a generalized $L S$ path $p$ with $p(0)=$ $\lambda, p(1)=\nu$ so that

1 .

$$
\text { length }_{\Delta}(p)=\left(\mu_{1}, \ldots, \mu_{m}\right),
$$

2.

$$
\mu_{i} \in \mathbb{Z}_{+} \varpi_{i}, \forall i=1, \ldots, m
$$


3.

$$
\sum_{i=1}^{m} \mu_{i}=\mu
$$

4. $p$ is contained in $\Delta$.

The following lemma easily follows from the above theorem, one can also derive it directly from the definition of a generalized LS path:

Lemma 2.10. If $p$ is a generalized LS path then

$$
p(0)-p(1)-\operatorname{length}_{\Delta}(p) \in Q(R) .
$$

2.6. The saturation theorem. In this section we discuss the Saturation Theorem proven in [13].

Recall that in [12] we have defined two constants $k_{R}$ and $k_{w}$ associated with the root system $R$. The saturation constant $k_{R}$ is defined by the property that it is the least integer $k \geq 1$ such that for each vertex $v$ of the Euclidean Coxeter complex $\left(A, W_{R, a f f}\right)$ we have:

$$
k v \in P(R),
$$

i.e. is a special vertex. The constant $k_{w}$ is the least integer $k \geq 1$ with the following property:

Suppose that $F$ is a face of $\left(A, W_{R, a f f}\right)$ invariant under an isometry of $A$ and let $v$ denote the barycenter of $F$. Then $k v$ is a special vertex.

We have proven in [12] that for the root systems $B_{\ell}$ and $C_{\ell}$ we have:

$$
k_{R}=k_{w}=2 .
$$

In particular, $k_{R}$ and $k_{w}$ do not change if we replace $R$ with $R^{\vee}$.

Let $\underline{G}$ be a reductive algebraic group over $\mathbb{Z}$; set $G=\underline{G}(\mathbb{C})$. For a nonarchimedean local field $\mathbb{K}$ (e.g. $\mathbb{K}=\mathbb{Q}_{p}$ ) we let $X$ denote the Bruhat-Tits building associated with $\underline{G}^{\vee}(\mathbb{K})$, where $\underline{G}^{\vee}$ is the Langlands' dual of $\underline{G}$.

Theorem 2.11. 1. Suppose that $\sigma=(\lambda, \mu, \nu) \in P(R)^{3} \cap \mathcal{P}(G)$ is such that $\lambda+\mu+\nu \in Q(R)$. Then

$$
k_{R}^{2} \cdot \sigma \in \operatorname{Tens}(G)
$$

2. Suppose that $\sigma=(\lambda, \mu, \nu) \in P(R)^{3} \cap \mathcal{P}(G)$. Then

$$
k_{R} k_{w} \cdot \sigma \in \operatorname{Tens}(G) .
$$

Proof. The first assertion is the Saturation Theorem 1.8 of [13]. We prove the second assertion.

Then, since $\sigma \in \mathcal{P}(G)=D_{3}(X)$, there exists a geodesic triangle $[z, x, y]$ in $X$ with the $\Delta$-side lengths $(\lambda, \mu, \nu)$ (see [11]). According to Theorem 7.16 of [12], there exists a geodesic triangle $\left[z^{\prime}, x^{\prime}, y^{\prime}\right] \subset X$ whose vertices are special vertices of $X$ and whose 
$\Delta$-side lengths are $\left(\lambda^{\prime}, \mu^{\prime}, \nu^{\prime}\right):=\left(k_{w} \lambda, k_{w} \mu, k_{w} \nu\right)$. Therefore, by Part 2 of Theorem 1.8 in [13],

$$
k_{R}\left(\lambda^{\prime}, \mu^{\prime}, \nu^{\prime}\right) \in \operatorname{Tens}(G)
$$

In section 5 we will need the following improvement of Theorem 2.11 in the case of the root system $R=B_{2} \cong C_{2}$.

TheOREM 2.12. Suppose that $G$ has the root system $R \cong B_{2}, \sigma=(\lambda, \mu, \nu) \in$ $P(R)^{3} \cap \mathcal{P}(G)$ and $\lambda+\mu+\nu \in Q(R)$. Then:

1 .

$$
2 \cdot \sigma \in \operatorname{Tens}(G)
$$

2. Moreover, there exists a generalized $L S$ path $p \in L S_{1}(R)$ contained in $\Delta$, connecting $2 \lambda$ to $2 \nu=2 \nu^{*}$, so that length $\Delta(p)=\left(2 \mu_{1}, 2 \mu_{2}\right), \mu_{1}+\mu_{2}=\mu, \mu_{i} \in \mathbb{Z}_{+} \varpi_{i}$ and all break-points of $p$, with possible exception of ones occurring on the boundary of $\Delta$, are special vertices.

Proof. The first assertion follows from the second. The proof of the second assertion is a variation on the proof of the Saturation Theorem 1.8 given in [13] so here we will give only a sketch and refer the reader to [13] for the details.

Let $X$ be the Euclidean building as above. Then the assumptions that $\sigma=$ $(\lambda, \mu, \nu) \in P(R)^{3} \cap \mathcal{P}(G)$ and $\lambda+\mu+\nu \in Q(R)$ imply that there exists a geodesic triangle $[\tilde{x}, \tilde{y}, \tilde{z}] \subset X$ whose vertices are vertices of $X$ and whose $\Delta$-side-lengths are $\mu, \nu, \lambda$, see [12, Corollary 7.12, Part 1]. We let $\tilde{A} \subset X$ denote an apartment containing the segment $\tilde{\tilde{x} \tilde{y}}$ and let $\tilde{\Delta} \subset \tilde{A}$ denote a translate of a Weyl chamber in $\tilde{A}$, so that the tip of $\tilde{\Delta}$ is at $\tilde{x}$, and $\overline{\tilde{x}} \tilde{y} \subset \tilde{\Delta}$. We identify the fundamental weights $\varpi_{1}, \varpi_{2}$ with vectors in $\tilde{A}$ so that $\mu=m_{1} \mu_{1}+m_{2} \mu_{2}$, where $\mu_{i}$ are multiples of $\varpi_{i}, i=1,2$. We then replace the segment $\overline{\tilde{x} \tilde{y}}$ with the concatenation

$$
\tilde{p}=\pi_{\mu_{1}} * \pi_{\mu_{2}} \subset \tilde{\Delta} .
$$

The path $\tilde{p}$ connects $\tilde{x}$ to $\tilde{y}$.

Lemma 2.13. Let $h: A \rightarrow A$ be a dilation by 2 which fixes a special vertex. Then the path $\tilde{p}$ crosses walls of $\tilde{A}$ transversally only at points $v$ such that $h(v)$ are special vertices.

Proof. To simplify the notation we identify the apartment $\tilde{A}$ with the model apartment $\left(A, W_{a f f}\right)$. Suppose first that $\tilde{x}$ is a special vertex. Since $\mu_{1} \in P(R)$, the end-point of the path $\pi_{\mu_{1}}$ is also a special vertex. Thus the paths $\pi_{\mu_{1}}, \pi_{\mu_{2}}$ (and therefore $\tilde{p}$ ) are entirely contained in the 1-dimensional skeleton of the simplicial complex $\left(A, W_{a f f}\right)$. Therefore these paths cross walls transversally only at the vertices of this complex. However $k_{R}=2$ for $R=C_{2}$ means that for each vertex $v \in\left(A, W_{a f f}\right)$, its image under dilation $h(v)$ is a special vertex. Hence the claim follows in this case.

Suppose that $\tilde{x}$ is not a special vertex. Nevertheless, this point is a vertex of an alcove $a \subset\left(A, W_{a f f}\right)$. The break-point $\tilde{u}$ of the path $\tilde{p}$ is also a vertex of $\left(A, W_{a f f}\right)$ which has the same type as $\tilde{x}$. Consider now a pair of points $u \in a, s \in A$ which are nonspecial vertices so that the geodesic segment $J=\overline{u s} \subset\left(A, W_{a f f}\right)$ is parallel to an element of $W \cdot \varpi_{1}$ or $W \cdot \varpi_{2}$. Then for each point $v$ of transversal intersection of $J$ with walls of $\left(A, W_{a f f}\right)$ we have: 
- Either the type $\theta(v)$ of $v$ is a vertex of $a$, in the case when $J$ is parallel to an element of $W \cdot \varpi_{2}$,

- Or the type $\theta(v)$ equals $\frac{1}{2} \varpi_{2}$, in the case when $J$ is parallel to an element of $W \cdot \varpi_{1}$, see Figure 1 .

In either case, $h(v)$ is again a special vertex of $\left(A, W_{a f f}\right)$. $\square$

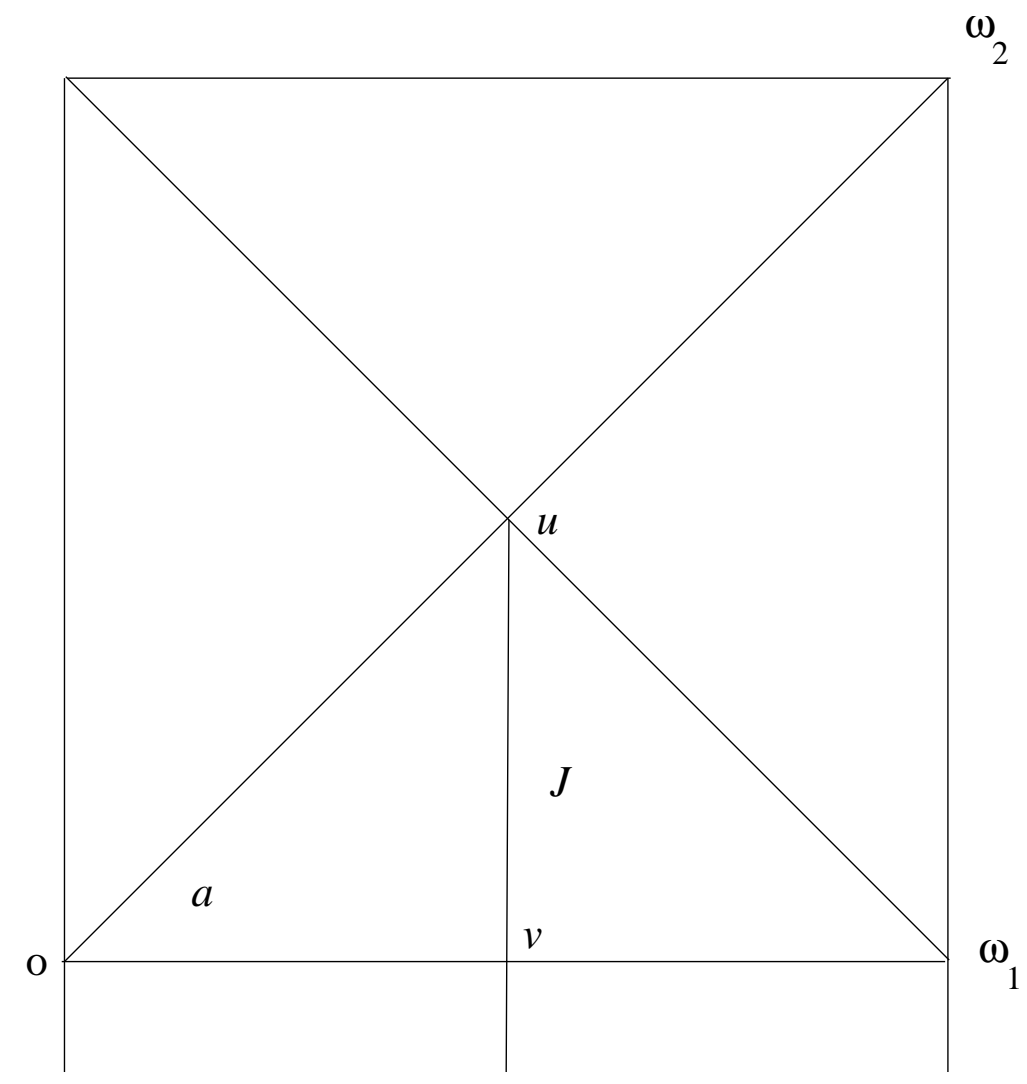

FIG. 1.

REMARK 2.14. The direct generalization of Lemma 2.13 fails in the case of the root system $B_{3}$ where the path $\tilde{p}$ is the concatenation of three geodesic segments parallel to the fundamental weights.

Recall that in [13] we have defined a family of projections $f=$ Fold $_{z, k, \Delta}: X \rightarrow \Delta$, where $\Delta \subset A$ is a Weyl chamber with tip at $o$. Here $k \in \mathbb{N}, z$ is a vertex in $A$. The mapping $f$ is the composition of three maps:

1. A retraction Fold $_{a, A}: X \rightarrow A$ with respect to an alcove $a$ containing $z$.

2. Dilation $h: A \rightarrow A$ by $k$ so that $h(z)=o$.

3. Projection $\mathbb{P}_{\Delta}: A \rightarrow \Delta$.

Then

$$
f=\mathbb{P}_{\Delta} \circ h \circ \text { Fold }_{a, A}
$$

Consider the path

$$
p:=\operatorname{Fold}_{z, 2, \Delta}(\tilde{p})
$$


where $z:=\tilde{z}$. It was shown in [13] that the path $p$ is in $L S_{1}(R)$ and

$$
\underline{\text { length }}_{\Delta}(p)=2 \underline{\text { length }}_{\Delta}(\tilde{p})=2\left(\mu_{1}, \mu_{2}\right) .
$$

It is clear that $p$ is contained in $\Delta$ and that this path connects $x:=f(\tilde{x})$ to $y:=f(\tilde{y})$ where $\overrightarrow{o x}=2 \lambda, \overrightarrow{o y}=2 \nu$. Observation 2.13 implies that all break-points of the path $q:=h \circ F_{o l d}, A(\tilde{p})$ are special vertices of $A$. Their images under the projection $\mathbb{P}_{\Delta}$ are also special. The projection $\mathbb{P}_{\Delta}$ may introduce new break-points in the path $p$ (i.e. break-points which are not projections of break-points of $q$ ). However such points necessarily belong to the walls of $\Delta$. $\square$

Remark 2.15. It is proven in Proposition 8.34 of [12] that in Part 1 of Theorem 2.12, the assumption that $\lambda+\mu+\nu \in Q(R)$ can be omitted. Theorem 5.3 proven in section 5.4 of the present paper, provides an alternative proof of this result.

\section{Decomposition of Tens $(G)$ into elementary subsets.}

Definition 3.1. Call a subset $E \subset \mathbb{Z}^{n}$ elementary if it is defined via a finite system of (non-strict, possibly inhomogeneous) linear inequalities with rational coefficients and congruences, i.e. equations of the form $h(x) \in \mathbb{Z}$, where $h$ is a rational linear function.

Note that each system of congruence conditions on $x$ is equivalent to the requirement that $x$ belongs to a coset of a subgroup in $\mathbb{Z}^{n}$. By adding a linear equation to the system of inequalities, we can reduce a system of congruence conditions on $x$ to the requirement that $x \in L+z$, where $L$ is a sublattice in $\mathbb{Z}^{n}$ (i.e. a rank $n$ subgroup) and $z$ is a certain element of $\mathbb{Z}^{n}$.

The next proposition follows for instance from [7, Theorem 1] (we are grateful to C. Laskowski for this reference), but we give an elementary proof for the sake of completeness.

Proposition 3.2. If $E \subset \mathbb{Z}^{n}$ is an elementary subset then its projection to $\mathbb{Z}^{n-1}$ is a finite union of elementary subsets.

Proof. We let $(x, a)$ denote coordinates in $\mathbb{Z}^{n}$ so that $x$ is the coordinate in $\mathbb{Z}^{n-1}$. Suppose that the elementary set $E$ is given by the linear inequalities

$$
F(x, a) \in\left(\mathbb{R}_{+}\right)^{m}
$$

and congruences $(x, a) \in L$, where $L$ is a translate of a sublattice in $\mathbb{Z}^{n}$. Therefore, up to changing coordinates via an integer translation, $L$ is a sublattice in $\mathbb{Z}^{n}$. Since $L$ has finite index in $\mathbb{Z}^{n}$, there exists an integer $\kappa$ so that $\kappa \cdot \mathbb{Z}^{n} \subset L$; therefore $L$ is a union of finitely many cosets

$$
L_{i}:=z_{i}+\kappa \cdot \mathbb{Z}^{n} .
$$

By restricting to the cosets $L_{i}$ and making the linear changes of variables $(x, a) \rightarrow$ $(x, a)-z_{i}$, we reduce the proof to the case when $L$ has the form $\kappa \cdot \mathbb{Z}^{n}$, which we assume from now on.

Let $\tilde{P}$ denote the convex polyhedron $\left\{(x, a): F(x, a) \in\left(\mathbb{R}_{+}\right)^{m}\right\}$. Let $P$ denote the projection of $\tilde{P}$ to $\mathbb{R}^{m-1}$ under the map $p:(x, a) \mapsto x$; this set is again a convex polyhedron. Then we can subdivide $P$ into a finite union of convex polyhedra $P_{i}$ 
(each given by a linear system of inequalities with rational coefficients) such that for each $i$ the set $p^{-1}\left(P_{i}\right) \cap \tilde{P}$ is given by the two inequalities

$$
g_{i}(x) \leq a \leq f_{i}(x)
$$

where $g_{i}, f_{i}$ are linear functions with rational coefficients. From now on we fix $i$ and set $f:=f_{i}, g:=g_{i}$ : We will show that $p(E) \cap P_{i}$ is a finite union of elementary sets.

Observe that the restriction of $f / \kappa$ to $\kappa \cdot \mathbb{Z}^{n-1}$ takes only finitely many values $(\bmod \mathbb{Z})$

$$
t_{j} \in[0,1), j=1, \ldots, J .
$$

Let $\Lambda_{j}$ denote the coset in $\kappa \mathbb{Z}^{n-1}$ such that the value of $f / \kappa$ on $\Lambda_{j}$ equals $t_{j}$ mod $\mathbb{Z}$. Let $[f(x) / \kappa]$ denote the integer part. Then the condition that there exists $a \in \kappa \mathbb{Z}$ such that

$$
g(x) \leq a \leq f(x)
$$

is equivalent to

$$
g(x) / \kappa \leq[f(x) / \kappa]
$$

i.e.

$$
g(x) / \kappa \leq f(x) / \kappa-t_{j}, x \in \Lambda_{j}
$$

Therefore the projection of $E \cap p^{-1}\left(P_{i}\right)$ to $P_{i}$ equals

$$
E_{i j}=\bigcup_{j=1}^{J}\left\{x \in P_{i} \cap \Lambda_{j}: g(x) \leq f(x)-\kappa t_{j}\right\} .
$$

It is clear that each $E_{i j}$ is an elementary set.

Our next goal is to show that the semigroup Tens $:=\operatorname{Tens}(G)$ is a finite union of elementary sets, where $G$ is a complex reductive Lie group. Let $L=X^{*}(T)$ denote the cocharacter lattice of a maximal torus $T \subset G$. We let $\varpi_{1}, \ldots, \varpi_{\ell}$ denote the fundamental weights of $G, \Pi:=W \cdot\left\{\varpi_{1}, \ldots, \varpi_{\ell}\right\}$, where $W$ is the Weyl group of $G$. Let $\alpha_{1}, \ldots, \alpha_{\ell}$ denote the simple roots in $R$. Let $\alpha_{i}^{\vee}$ denote the coroots.

For each $\eta \in \Pi$ let $\iota(\eta)$ denote the number $i$ such that $\eta \in W \cdot \varpi_{i}$. Let $\Sigma$ denote the set of generalized chains $\sigma$ in $\Pi$, i.e. sequences

$$
\eta_{0} \gtrsim \eta_{1} \gtrsim \ldots \gtrsim \eta_{m}
$$

of elements of $\Pi$ so that

$$
\iota\left(\eta_{0}\right) \leq \iota\left(\eta_{1}\right) \leq \ldots \leq \iota\left(\eta_{m}\right)
$$

Let $\Sigma_{\max }$ denote the collection of maximal generalized chains as above, i.e. chains of maximal length from $\eta_{0}$ to $\eta_{m}$.

Let $\lambda, \mu, \nu \in \Delta \cap L$ be dominant characters,

$$
\mu=\sum_{i=1}^{\ell} n_{i} \varpi_{i} .
$$


We consider broken geodesic paths $\pi:[0,1] \rightarrow V=P(R) \otimes \mathbb{R}$ modeled on the chain $\sigma$ as above, with $p(0)=0$, i.e. concatenations of paths

$$
\pi_{a_{i} \eta_{i}}, i=0, \ldots, m
$$

where $a_{i} \in \frac{1}{k_{R}^{2}} \mathbb{Z}, a_{i} \geq 0$. We require that

$$
\underline{\text { length }}_{\Delta}(\pi)=\left(n_{1} \varpi_{1}, \ldots, n_{\ell} \varpi_{\ell}\right) .
$$

This means that

$$
a_{1}+\ldots+a_{J_{1}}=n_{1}, a_{J_{1}+1}+\ldots+a_{J_{2}+2}=n_{2}, \ldots
$$

We define the partial sums

$$
S_{r}=\sum_{j=1}^{r} a_{j} \eta_{j}
$$

for $1 \leq r \leq m$. Observe that $\iota\left(\eta_{r}\right)=\iota\left(\eta_{r+1}\right)$ if nd only if there exists a reflection $\tau_{r}=\tau_{\alpha_{r}^{\vee}}$ which carries $\eta_{r}$ to $\eta_{r+1}$. Then the path $\pi$ is a generalized LS path if and only if the following condition is satisfied:

For each $r$ such that $\iota\left(\eta_{r}\right)=\iota\left(\eta_{r+1}\right)$ we have

$$
\alpha_{r}^{\vee}\left(S_{r}\right)=\sum_{j=1}^{r} a_{j} \alpha_{r}^{\vee}\left(\eta_{j}\right) \in \mathbb{Z} .
$$

Note that these conditions imply that if $\iota\left(\eta_{r}\right) \neq \iota\left(\eta_{r+1}\right)$ then $S_{r}$ is necessarily a special vertex, i.e. all coroots take integer values at this point.

Since $\alpha_{r}^{\vee}\left(\eta_{j}\right) \in \mathbb{Z}$ for all $r, j$, it follows that the above integrality condition is a congruence condition on the $m$-tuple $a=\left(a_{0}, \ldots, a_{m}\right)$.

According to Theorem 2.9, we have: $(\lambda, \mu, \nu) \in \operatorname{Tens}(G)$ if and only if there exists a generalized LS path $\pi$ as above so that:

(1) $\lambda+\pi(1)=\nu^{*}$.

(2) The entire path $\lambda+\pi(t)$ is contained in the positive chamber $\Delta$, i.e. for each simple coroot $\alpha^{\vee}$ and each partial sum

$$
S_{r}=\sum_{j=1}^{r} a_{j} \eta_{j}
$$

we have:

$$
\alpha^{\vee}\left(\lambda+S_{r}\right) \geq 0
$$

Let $\lambda=\sum_{i=1}^{\ell} m_{i} \varpi_{i}, \nu=\sum_{i=1}^{\ell} k_{i} \varpi_{i}$. Set

$$
x:=\left(m_{1}, \ldots, m_{\ell}, n_{1}, \ldots, n_{\ell}, k_{1}, \ldots, k_{\ell}\right) .
$$

Note that the condition (1) is a linear equation with integer coefficients on the vector $a$ and the condition (2) has the form of a system of linear inequalities with integer coefficients. Therefore for each generalized chain $\sigma$ as above, the set

$$
E_{\sigma}:=\left\{(x, a): \lambda+p(1)=\gamma^{*}, \alpha^{\vee}\left(\lambda+S_{r}\right) \geq 0, \forall \alpha \in \Phi, \text { and } \alpha_{r}^{\vee}\left(S_{r}\right) \in \mathbb{Z}, r=0, \ldots, m-1\right\}
$$


is an elementary set.

REMARK 3.3. Instead of using path model in the above argument one can use the polytopal model from [3].

Consider the projection $p\left(E_{\sigma}\right)$ of $E_{\sigma}$ to the $x$-coordinate. By applying Proposition 3.2 inductively we conclude that $p\left(E_{\sigma}\right)$ is a finite union of elementary sets. Therefore the union

$$
\operatorname{Tens}(G)=\bigcup_{\sigma \in \Sigma_{\max }} p\left(E_{\sigma}\right)
$$

is also a finite union of elementary sets.

Thus we have proved the following analogue of Laskowski's theorem in [17]:

THEOREM 3.4. The semigroup Tens $(G)$ is a finite union of elementary sets.

4. Deep subcone. The goal of this section is to show that deep inside of the cone $D_{3}=\mathcal{P}(G)$ there is a subcone of the form $D_{3}+\sigma$ (for some $\sigma \in D_{3}$ ), such that

$$
\left(D_{3}+\sigma\right) \cap \Lambda \subset \operatorname{Tens}(G) .
$$

We will also present an explicit computation of this subcone for the root system $B_{\ell}$. In what follows we normalize the roots so that the Euclidean norm of each coroot is either 1 or 2 .

Given an irreducible representation $V_{\lambda}$ let $W$ eight $\left(V_{\lambda}\right) \subset P(R)$ denote the set of weights of $V_{\lambda}$. Define the set

$$
S_{3}(G)^{1,2}:=\left\{(\lambda, \mu, \nu) \in \Delta^{3}: \lambda \gg \mu, \nu^{*}=\lambda+\beta \text {, for some } \beta \in W \operatorname{eight}\left(V_{\mu}\right)\right\} .
$$

Here $\lambda \gg \mu$ iff $\lambda+\beta \in \Delta$ for all $\beta \in W$ eight $\left(V_{\mu}\right)$.

Below we give a more explicit description of the above subset $S_{3}(G)^{1,2}$ in terms of linear inequalities and congruences.

We will be using the following notation: If $C \subset V$ is a convex subset then $\lambda \geq_{C} \mu$ iff $\mu-\lambda \in C$. We use the notation $\Delta^{*}$ for the convex cone dual to $\Delta$, i.e.

$$
\Delta^{*}=\{v \in V: v \cdot u \geq 0, \forall u \in V\}
$$

Define the lattice

$$
\Lambda=\{(\mu, \nu, \lambda): \mu, \nu, \lambda \in L, \lambda+\mu+\nu \in Q(R)\},
$$

where $L=X^{*}(T)$. We let $C^{1,2}$ be convex polyhedral cone in $\Delta^{3}$ given by the following inequalities:

1.

$$
w \mu^{*} \leq_{\Delta} \lambda, \quad \forall w \in W
$$

2.

$$
w \nu^{*} \leq_{\Delta^{*}} w \lambda+\mu, \quad \forall w \in W .
$$

Proposition 4.1.

$$
S_{3}(G)^{1,2}=C^{1,2} \cap \Lambda .
$$


Proof.

LEMMA 4.2 .

$$
\lambda \gg \mu \Longleftrightarrow w \mu^{*} \leq_{\Delta} \lambda, \quad \forall w \in W .
$$

Proof. First let's check that if $\lambda+w \mu \in \Delta$ for all $w \in W$ then

$$
\lambda+\beta \in \Delta, \forall \beta \in W \operatorname{eight}\left(V_{\mu}\right) .
$$

Indeed, let $\beta \in W e i g h t\left(V_{\mu}\right)$. We write $\beta$ as a convex combination of extreme weights $\{w \mu, w \in W\}:$

$$
\beta=\sum_{w \in W} t_{w}(w \mu), \sum_{w \in W} t_{w}=1,0 \leq t_{w} \leq 1
$$

Then

$$
\lambda+\beta=\lambda+\sum_{w \in W} t_{w}(w \mu)=\sum_{w \in W} t_{w} \lambda+\sum_{w \in W} t_{w}(w \mu)=\sum_{w \in W} t_{w}(\lambda+w \mu) .
$$

By assumption, $\lambda+w \mu \in \Delta$. Since $\Delta$ is convex, $\lambda+\beta \in \Delta$ as well. Therefore

$$
\lambda \gg \mu \Longleftrightarrow \lambda+w \mu \geq_{\Delta} 0, \forall w \in W \Longleftrightarrow \lambda+w w_{0} \mu \geq_{\Delta} 0, \forall w \in W,
$$

where $w_{0} \in W$ is the longest element.

$$
\lambda+w w_{0} \mu \geq_{\Delta} 0 \Longleftrightarrow w w_{0}(-\mu) \leq_{\Delta} \lambda \Longleftrightarrow w \mu^{*} \leq_{\Delta} \lambda,
$$

since $\mu^{*}=w_{0}(-\mu)$.

We refer the reader to [9] for the proof of the following:

Lemma 4.3. For a dominant weight $\mu$ and a weight $\beta$ we have

$$
\beta \in W \text { eight }\left(V_{\mu}\right) \Longleftrightarrow w \beta \leq_{\Delta^{*}} \mu, \quad \forall w \in W, \text { and } \mu-\beta \in Q(R) \text {. }
$$

Lemma 4.4. Let $\lambda, \mu, \nu$ be dominant weights. Then

$$
\nu^{*}=\lambda+\beta, \text { for some } \beta \in W \operatorname{eight}\left(V_{\mu}\right)
$$

if and only if

$$
w \nu^{*} \leq_{\Delta^{*}} w \lambda+\mu, \text { and } \mu-\nu^{*}+\lambda \in Q(R)
$$

Proof.

$\nu^{*}-\lambda \in W \operatorname{eight}\left(V_{\mu}\right) \Longleftrightarrow w\left(\nu^{*}-\lambda\right) \leq_{\Delta^{*}} \mu, \forall w \in W$, and $\mu-\left(\nu^{*}-\lambda\right) \in Q(R)$.

It remains to prove 
LEMMA 4.5.

$$
\mu-\nu^{*}+\lambda \in Q(R) \Longleftrightarrow \mu+\nu+\lambda \in Q(R) .
$$

Proof. It suffices to prove that $\mu-\nu^{*}+\lambda-(\mu+\nu+\lambda) \in Q(R)$, equivalently,

$$
\nu+\nu^{*}=\nu-w_{0} \nu \in Q(R) .
$$

However for each $\nu \in P(R), w \in W$ we have $w \nu \in W$ eight $\left(V_{\nu}\right)$, therefore

$$
\nu-w \nu \in Q(R)
$$

by Lemma 4.3 .

This concludes the proof of Proposition 4.1.

The following result is standard, we are grateful to T. Haines and S. Kumar for pointing out this result to us and explaining the proofs:

TheOrem 4.6. The subset $S_{3}(X)^{1,2}$ is contained in Tens $(G)$.

Proof. We will present the proof of this result using Littelmann's path model.

We need the following

Lemma 4.7. Suppose that $p$ is an LS path of $\Delta$-length $\mu$. Then the path $p$ is entirely contained in the convex hull of the $W$-orbit $S:=W(\mu)$.

Proof. By definition of an LS path, for each $t \in[0,1]$ there exist $t_{i} \geq 0$ and $\nu_{i} \in W \mu$ such that

$$
t=t_{1}+\ldots+t_{j}
$$

and

$$
p(t)=t_{1} \nu_{1}+\ldots+t_{j} \nu_{j} .
$$

Therefore, since $t \in[0,1]$, and the convex hull of $S$ contains the origin, the subconvex combination $p(t)$ is contained in the convex hull of $S$.

Suppose now that $\sigma=(\lambda, \mu, \nu) \in S_{3}(X)^{1,2}$. Then $\nu^{*}=\lambda+\beta$ for some weight vector $\beta$ of the representation $V_{\mu}$. Then, according to [18], there exists an LS path $p$ of the $\Delta$-length $\mu$ such that $p(0)=0, p(1)=\beta$. By the above Lemma, the path $p$ is entirely contained in the convex hull of $W \mu$. Consider the path $q(t):=\lambda+p(t)$. We claim that this path is entirely contained in $\Delta$. Indeed,

$$
q(t) \in \lambda+\operatorname{Hull}(W \mu) .
$$

Since $\lambda \gg \mu$, for each vector $\gamma \in W \cdot \mu$, we have:

$$
\lambda+\gamma \in \Delta \text {. }
$$

Thus $\lambda+H u l l(W \mu) \subset \Delta$. On the other hand, $q(1)=\lambda+\beta=\nu^{*}$. Therefore, according to Theorem $2.9,(\lambda, \mu, \nu) \in$ Tens $(G)$. 
We next observe that the cone $C^{1,2}$ has nonempty interior. Indeed, first choose $\mu \in \operatorname{Int}(\Delta)$. Then take $\lambda \in \Delta$ such that

$$
d(\lambda, \partial \Delta)>|\mu| .
$$

Finally, pick $\nu^{*}$ sufficiently close to $\lambda$ so that

$$
\left|\lambda-\nu^{*}\right|<d\left(\mu, \partial \Delta^{*}\right)
$$

Any triple $(\lambda, \mu, \nu)$ chosen like this satisfies the strict inequalities

$$
\begin{gathered}
w \mu^{*}<_{\Delta} \lambda, \quad \forall w \in W, \\
w \nu^{*}<_{\Delta^{*}} w \lambda+\mu, \quad \forall w \in W .
\end{gathered}
$$

and therefore belongs to the interior of $C^{1,2}$. Our next goal is to apply the above observations to show that the cone $\mathcal{P}(G)$ contains a subcone $\sigma+\mathcal{P}(G)$ such that

$$
\sigma+\mathcal{P}(G) \cap \Lambda=\operatorname{Tens}(G) \cap \Lambda .
$$

Since $C^{1,2}$ is a homogeneous cone with nonempty interior, it contains metric balls $B\left(\sigma_{0}, R\right)$ of arbitrarily large radius $R$. Let $k:=k_{R} k_{w}$. Choose $R$ larger than the diameter of a fundamental domain $F$ for the lattice $k \cdot P(R)$. (Here $F$ is a certain fundamental parallelepiped containing the origin.) Without loss of generality we assume that the ball $B\left(\sigma_{0}, R\right)$ is centered at a point $\sigma_{0} \in k \cdot P(R)$.

Suppose now that $\tau \in \Lambda \cap D_{3}(X)$. Then there exists a point $\sigma \in k \cdot P(R)$ such that $\tau \in F+\sigma$. Let $\kappa:=\sigma-\sigma_{0}$. Since

$$
F+\sigma=\left(F+\sigma_{0}\right)+\kappa,
$$

there exists a point $\tau_{0} \in F+\sigma_{0}$ such that $\tau=\tau_{0}+\kappa$.

Note that $k \cdot P(R) \subset Q(R)$, since the index $|P(R): Q(R)|$ divides $k_{R} k_{w}$, see [12], Table 11. Therefore

$$
\sigma_{0} \in k \cdot P(R)^{3} \subset \Lambda
$$

and hence $\tau_{0}=\tau-\kappa=\tau-\sigma+\sigma_{0} \in \Lambda$. Since $\operatorname{diam}(F) \leq R, F+\sigma_{0}$ is contained in $B\left(\sigma_{0}, R\right) \subset C^{1,2}$. Therefore, by Theorem 4.6, $\tau_{0} \in F+\sigma_{0} \subset C^{1,2} \subset T e n s(G)$. By the triangle inequality, if

$$
d\left(\tau, \partial D_{3}(X)\right) \geq\left|\sigma_{0}\right|+\operatorname{diam}(F),
$$

then $\kappa=\sigma-\sigma_{0}$ belongs to $\mathcal{P}(G)$. On the other hand, since $\sigma, \sigma_{0} \in k \cdot P(R)$, it follows that $\kappa \in k \cdot P(R)$ and therefore, by the saturation theorem,

$$
\kappa \in \operatorname{Tens}(G) .
$$

Therefore, since $\tau_{0} \in \operatorname{Tens}(G)$ and $\operatorname{Tens}(G)$ is a semigroup, it follows that

$$
\tau=\tau_{0}+\kappa
$$

also belongs to Tens $(G)$. Hence we have proven 
TheOREM 4.8. Suppose that $\tau \in \mathcal{P}(G) \cap \Lambda$ is such that $d(\tau, \partial \mathcal{P}(G)) \geq\left|\sigma_{0}\right|+$ $\operatorname{diam}(F)$. Then $\tau \in \operatorname{Tens}(G)$.

Next, the linear inequalities defining $\mathcal{P}(G)$ have the form

$$
\psi_{j}(x) \geq 0,
$$

where either $\left|\psi_{j}\right| \leq 2$ (in the case of the inequalities $\psi_{j} \geq 0$ defining the chamber $\Delta$ ) or $\left|\psi_{j}\right|=\sqrt{3}\left|\varpi_{i_{j}}\right|$ for a certain fundamental weight $\varpi_{i_{j}}$ (in the case of the stability inequalities). Here we are using the Euclidean norm of linear functionals. Set $m:=$ $\max _{i}\left|\varpi_{i}\right|$ and note that, because of our normalization of the lengths of the coroots, $m \geq 2$. Therefore,

$$
\max _{j}\left|\psi_{j}\right|=m
$$

Suppose that $\tau-\kappa \in \partial \mathcal{P}(G)$. Then there exists some $\psi_{j}$ such that $\psi_{j}(\tau-\kappa)=0$, i.e.

$$
\left|\psi_{j}(\tau)\right|=\left|\psi_{j}(\kappa)\right| \leq\left|\psi_{j}\right| \cdot|\kappa| \leq m|\kappa|
$$

and $|\kappa| \geq\left|\psi_{j}(\tau)\right| / m$. Thus, if

$$
\psi_{j}(\tau) \geq m\left(\left|\sigma_{0}\right|+\operatorname{diam}(F)\right), \forall j,
$$

then $d(\tau, \partial \mathcal{P}(G)) \geq\left|\sigma_{0}\right|+\operatorname{diam}(F)$. Therefore, if we define an inhomogeneous subcone $\mathcal{P}^{\prime}(G)$ in $\mathcal{P}(G)$ by the linear inequalities

$$
\psi_{j}(\tau) \geq m\left(\left|\sigma_{0}\right|+\operatorname{diam}(F)\right), \forall j,
$$

we obtain:

Corollary 4.9. The intersection $\mathcal{P}^{\prime}(G) \cap \Lambda$ is contained in Tens $(G)$.

Below we make an explicit computation for the root system $R=B_{\ell}$ (i.e. when $G=S p(2 \ell, \mathbb{C}))$. We use the Bourbaki coordinates [4], to describe this root system. Since for this root system $w_{0}=-1$, it follows that $\lambda=\lambda^{*}$ for each $\lambda \in \Delta$. We let $\left\{\epsilon_{i}\right\}$ denote the standard orthonormal basis in $V$. Then for each $i, \varpi_{i}=\sum_{j=1}^{i} \epsilon_{i}$. Moreover, the simple roots are $\alpha_{i}=\epsilon_{i-1}-\epsilon_{i}, i<\ell$ and $\alpha_{\ell}=\epsilon_{\ell}$. The positive chamber $\Delta$ is given by the inequalities $\alpha_{i} \cdot v \geq 0, i=1, \ldots, \ell$.

Theorem 4.10. Suppose that $R=B_{\ell}(\ell \geq 2)$, and $\tau \in \Lambda$ is such that for each linear functional $\psi$ which appears in the system of stability inequalities and chamber inequalities we have:

$$
\psi(\tau) \geq 2 \ell^{2}(\ell+1)(4 \ell+5)+6 \ell .
$$

Then $\tau \in \operatorname{Tens}(\operatorname{Sp}(2 \ell, \mathbb{C}))$.

Proof. We will use the notation from the proof of Theorem 4.8. In order for $\tau$ to be in $\operatorname{Tens}(S p(2 \ell, \mathbb{C}))$ we need two things:

$$
\sigma_{0}+F \subset C^{1,2}
$$

and

$$
\kappa=\tau-\tau_{0} \in \mathcal{P}(G), \quad \forall \tau_{0} \in F+\sigma_{0}
$$


We simplify the discussion by observing that in the case of the root system $B_{\ell}$ we have $k=k_{R}=k_{w}=k_{G}=2$ (using the notation of [12]), which means that instead of working with the lattice $\Lambda$ we can work with the lattice $L^{3}$, where $L=P\left(R^{\vee}\right)$ :

According to Part 2 of Theorem 2.11, for each $\sigma \in L^{3} \cap \mathcal{P}(G)$, the vector $k^{2} \sigma$ belongs to Tens $(G)$.

The lattice $L$ is just the integer lattice in $\mathbb{R}^{\ell}$ (using Bourbaki coordinates). Therefore we choose the fundamental domain $F$ for the sublattice $(4 L)^{3}$ to be the cube whose edges have length 4 and which is centered at the origin.

The condition (5) would follow from:

$$
\psi_{j}(\tau) \geq \psi_{j}\left(\tau_{0}\right)
$$

which in turn is implied by

$$
\psi_{j}(\tau) \geq \psi_{j}\left(\sigma_{0}\right)+\max _{f \in F}\left\{\psi_{j}(f)\right\}
$$

REMARK 4.11. $M=\max _{j, f \in F}\left\{\psi_{j}(f)\right\}$ equals the maximum of all $\psi_{j}$ 's on the set of points in $V^{3}$ with coordinates $\in\{0, \pm 1, \pm 2\}$. Here $V=P(R) \otimes \mathbb{R}$

In what follows we will use the norm

$$
\left\|\left(v_{1}, \ldots, v_{\ell}\right)\right\|=\sum_{i=1}^{\ell}\left|v_{i}\right|
$$

for vectors $v \in V$.

For the linear functionals $\psi_{j}$ which come from the stability inequalities, the maximum

$$
\max _{f \in F} \psi_{j}(f)
$$

does not exceed $6\left\|\varpi_{\ell}\right\|=6 \ell$. If the inequality $\psi_{j} \geq 0$ is one of the chamber inequalities, then $\psi_{j}(f) \leq 4 \leq 6 \ell$ for all $f \in F$. Thus $M \leq 6 \ell$.

Hence for the root system $B_{\ell}$ to guarantee (6) (and hence (5)) it suffices to require that

$$
\psi_{j}(\tau) \geq \psi_{j}\left(\sigma_{0}\right)+6 \ell, \forall j
$$
Set

To get an explicit estimate we have to choose an appropriate $\sigma_{0}=\left(\lambda_{0}, \mu_{0}, \nu_{0}\right)$.

$$
\mu_{0}:=(4 \ell, \ldots, 8,4)
$$

$a:=4 \ell+8, s:=8 \ell+4$ and

$$
\nu_{0}=\lambda_{0}:=(a+(\ell-1) s, \ldots, a+2 s, a+s, a) .
$$

Clearly $\lambda_{0}, \mu_{0}, \nu_{0} \in \Delta \cap 4 P(R)$.

Observe that

$$
\left\|\mu_{0}\right\|=2 \ell(\ell+1),
$$




$$
\left\|\lambda_{0}\right\|=\left\|\nu_{0}\right\|=a \ell+s \frac{\ell(\ell-1)}{2}=\ell\left(a+\frac{s \ell-s}{2}\right)=\ell\left(4 \ell^{2}+2 \ell+6\right) \leq 4 \ell(\ell+1)^{2} .
$$

Therefore for each linear functional $\psi$ of the form

$$
\psi(\lambda, \mu, \nu)=w_{1} \lambda \cdot \varpi_{i}+w_{2} \mu \cdot \varpi_{i}+w_{3} \nu \cdot \varpi_{i}
$$

we obtain:

$$
\left|\psi\left(\sigma_{0}\right)\right| \leq\left|\varpi_{i}\right|\left(\left\|\lambda_{0}\right\|+\left\|\mu_{0}\right\|+\left\|\nu_{0}\right\|\right) \leq \ell\left(8 \ell(\ell+1)^{2}+2 \ell(\ell+1)\right)=2 \ell^{2}(\ell+1)(4 \ell+5) .
$$

Therefore (7) follows from

$$
\psi(\tau) \geq 2 \ell^{2}(\ell+1)(4 \ell+5)+6 \ell, \forall \psi .
$$

We now consider the condition (4), i.e. that each

$$
(\lambda, \mu, \nu)=\sigma_{0}+f, f \in F,
$$

satisfies

$$
w \mu \leq_{\Delta} \lambda, \forall w \in W
$$

and

$$
w \nu \leq_{\Delta *} w \lambda+\mu, \forall w \in W .
$$

Let $\phi_{i j}$ denote the linear functionals of the form

$$
\phi_{i j}(\lambda, \mu, \nu)=\alpha_{i} \cdot\left(\lambda-w_{j} \mu\right)
$$

where $\alpha_{i}$ is a simple root and $w_{j} \in W$.

Let $\eta_{i j}$ denote the linear functionals of the form

$$
\eta_{i j}(\lambda, \mu, \nu)=\varpi_{i} \cdot\left(w_{j} \lambda+\mu-w_{j} \nu\right), w_{j} \in W .
$$

Then to guarantee (9) and (10) we need:

$$
\phi_{i j}\left(\sigma_{0}\right) \geq \phi_{i j}(f), \forall f \in F,
$$

and

$$
\eta_{i j}\left(\sigma_{0}\right) \geq \eta_{i j}(f), \forall f \in F
$$

respectively.

We first consider (12) since it is simpler. Since $\nu_{0}=\lambda_{0}$, the inequality (12) reads as

$$
\varpi_{i} \cdot \mu_{0} \geq \eta_{i j}(f), \forall f \in F .
$$

The right hand side of this inequality is $\leq 6 i$, while the left hand-side equals

$$
2(\ell+i)(\ell+1-i)
$$

It is clear that for $1 \leq i \leq \ell$ and each $\ell \geq 2$ we have:

$$
2(\ell+i)(\ell+1-i) \geq 2\left(\ell^{2}-i^{2}\right)+2(\ell+1)(\ell+i) \geq 6(\ell+i) \geq 6 i .
$$


This takes care of the condition (12).

Now consider (11). First note that $\phi_{i j}(f) \leq 8$ for all $i, j$ and $f \in F$. Next, for all $w \in W$ we have

$$
\alpha_{i} \cdot\left(w \mu_{0}\right) \leq 8 \ell-4
$$

for each $i=1, \ldots, \ell-1$ and

$$
\alpha_{\ell} \cdot\left(w \mu_{0}\right) \leq 4 \ell
$$

On the other hand,

$$
\phi_{i j}\left(\sigma_{0}\right)=\alpha_{i} \cdot\left(\lambda_{0}-w_{j} \mu_{0}\right)=\alpha_{i} \cdot \lambda_{0}-\alpha_{i} \cdot\left(w_{j} \mu_{0}\right) .
$$

We have:

$$
\alpha_{i} \cdot \lambda_{0}=s, \forall i<\ell
$$

and

$$
\alpha_{\ell} \cdot \lambda_{0}=a .
$$

Therefore for $i=1, \ldots, \ell-1$,

$$
\phi_{i j}\left(\sigma_{0}\right)-\phi_{i j}(f) \geq s-8 \ell+4-8=(8 \ell+4)-8 \ell-4=0
$$

and for $i=\ell$

$$
\phi_{i j}\left(\sigma_{0}\right)-\phi_{i j}(f) \geq a-(4 \ell+8)=(4 \ell+8)-(4 \ell+8)=0 .
$$

Therefore the condition (11) holds. Hence the inequality (8) implies that $\tau \in$ $\operatorname{Tens}(\operatorname{Sp}(2 \ell, \mathbb{C}))$.

Conjecture 4.12. Suppose that $G$ is a complex semisimple Lie group, $S$ is a stratum in the boundary of the cone $\mathcal{P}(G)$ which contains a point $(\lambda, \mu, \nu)$ such that all the vectors $\lambda, \mu, \nu$ are regular. Then there exists a subcone $S+\sigma \subset S$ such that

$$
S+\sigma \cap \Lambda=(S+\sigma) \cap \operatorname{Tens}(G) .
$$

5. Computation of the semigroup $\operatorname{Tens}(\operatorname{Sp}(4, \mathbb{C}))$. Let $V$ be $\mathbb{R}^{2}$ with the coordinates $x, y$ and Euclidean metric. Let $A$ denote the underlying affine space. Let $R:=C_{2} \subset V$ and $R^{\vee}=B_{2} \subset V^{*}=V$ be root systems with the sets of simple roots equal to $\left\{\alpha_{1}(x, y)=x-y, \alpha_{2}(x, y)=y\right\}$ and $\left\{\alpha_{1}^{\vee}(x, y)=x-y, \alpha_{2}^{\vee}(x, y)=2 y\right\}$ respectively. The fundamental weights of $C_{2}$ are $\varpi_{1}=(1,0), \varpi_{2}=(1,1)$; the root lattice is

$$
Q(R)=\left\{(x, y) \in \mathbb{Z}^{2}: x+y \in 2 \mathbb{Z}\right\}
$$

and the weight lattice is $P(R)=\mathbb{Z}^{2}$.

Let $W_{a f f}:=W_{R^{\vee}, a f f}, W_{a f f}^{\vee}:=W_{R, a f f}$ denote the affine Weyl groups acting on $A$ corresponding to the root systems $R$ and $R^{\vee}$ respectively. Note that $W_{a f f} \subset W_{a f f}^{\vee}$ is a subgroup of index 2 , so that all vertices of $\left(A, W_{a f f}\right)$ are special vertices of $\left(A, W_{a f f}^{\vee}\right)$. 
The root lattice $Q(R)$ equals the translation subgroup of $W_{\text {aff }}$ and the weight lattice $P(R)$ is the normalizer of $W_{a f f}$ in the group of Euclidean translations of $A$.

Our goal is to compute the semigroup Tens $=$ Tens $(R)$, for $R=C_{2}$. We know (from [12]) that each triple $\sigma=(\lambda, \mu, \nu) \in$ Tens satisfies:

1. $\sigma \in D_{3}:=D_{3}\left(C_{2}\right)$.

2. $\sigma \in P(R)^{3}$.

3. $\lambda+\mu+\nu \in Q(R)$.

It was shown in [12] that these conditions are necessary but not sufficient for $\sigma$ to be in Tens. The goal of this chapter to find necessary and sufficient conditions.

We start by observing that since $\lambda+\mu+\nu \in Q(R)$, it follows that at least one of the vectors $\lambda, \mu, \nu$ belongs to $Q(R)$.

Convention 5.1. Throughout the rest of the paper we will assume that $\mu \in$ $Q(R)$.

Notation 5.2. We break the vector $\mu$ as $\mu=\mu_{1}+\mu_{2}$, where $\mu_{i}=n_{i} \varpi_{i}$,

$$
\mu_{1}=\left(n_{1}, 0\right), \mu_{2}=\left(n_{2}, n_{2}\right), \mu=\left(n_{1}+n_{2}, n_{2}\right) .
$$

Since $\mu, \mu_{2} \in Q(R)$, it follows that $\mu_{1} \in Q(R)$ as well, i.e. $n_{1}$ is even.

Theorem 5.3. Suppose that $\sigma \in D_{3}(R) \cap P(R)^{3}$ is such that $\lambda+\mu+\nu \in Q(R)$. Then $\sigma \notin$ Tens if and only if two of the three vectors $\lambda, \mu, \nu$ belong to $\mathbb{Z}_{+} \varpi_{2}$ and $\lambda+\mu+\nu \notin 2 P(R)$.

Proof. The proof of this theorem occupies the rest of this chapter. Our strategy is to analyze the geometry of generalized LS paths with respect to the root system $2 R$ and show that all such paths (subject to the condition on $\lambda, \mu, \nu$ described in the above theorem) can be transformed to generalized LS paths with respect to the root system $R$. We first do this in the case of singular paths $p_{i} \in L S(2 R)$ with length ${ }_{\Delta}\left(p_{i}\right) \in \mathbb{N} \varpi_{i}$ (section 5.2) and then use the results to deal with the general $L S_{1}(2 R)$ paths $p=p_{1} * p_{2}$ (section 5.3). We are able to carry out this approach largely thanks to the fact that the root system $C_{2}$ is rather small and there are not that many chains formed by elements of $W \cdot \varpi_{i}, i=1,2$. This allows us to describe the paths in $L S_{1}(2 R)$ rather explicitly. A large supply of $L S_{1}(2 R)$ paths is given by the Saturation Theorem 2.12 which is another key tool in the proof.

To get an idea how the proof of Theorem 5.3 might proceed, consider the case when $\lambda, \mu, \nu \in Q(R)$ (i.e. the problem of decomposing tensor products of representations of $P S p(4, \mathbb{C}))$. Then $\lambda, \mu, \nu \in 2 P\left(R^{\vee}\right)$ and, according to Proposition 8.34 of $[12]$

$$
(\lambda, \mu, \nu) \in \operatorname{Tens}\left(R^{\vee}\right) .
$$

Therefore there exists a path $p \in L S_{1}\left(R^{\vee}\right)$ contained in $\Delta$ connecting $\lambda$ to $\nu$ and having

$$
\underline{\text { length }}_{\Delta}(p)=\left(\mu_{1}, \mu_{2}\right) \text {. }
$$

If we are lucky, all break-points of $p$ occur at special vertices of $\left(A, W_{a f f}^{\vee}\right)$ (compare Theorem 2.12), hence they are at the vertices of $\left(A, W_{a f f}\right)$, see Corollary 5.10. The key then is to replace $p$ with a new $L S_{1}\left(R^{\vee}\right)$ path $\hat{p}$ contained in $\Delta$ whose break-points occur at the special vertices of $\left(A, W_{a f f}\right)$, the crucial lemmas proving this are Lemmas $5.13,5.14$. This would imply that $\hat{p}$ is an $L S_{1}(R)$ path and $(\lambda, \mu, \nu) \in \operatorname{Tens}(R)$. 
5.1. Analysis of the $L S_{1}(R)$ paths. In this section we describe generalized LS paths $p$ with respect to the root system $R$. Recall that every such path is a concatenation

$$
p=p_{1} * p_{2}, \quad \operatorname{length}_{\Delta}\left(p_{i}\right) \in \mathbb{N} \varpi_{i}, i=1,2,
$$

where each $p_{i}$ is an LS path. Let $\tau_{i}$ denote the reflections in the walls $\left\{\alpha_{i}=0\right\}$, $i=1,2$.

Suppose that $p$ is a PL path in $A$ parameterized with the constant speed. We say that $t($ or $p(t))$ is a break-point of $p$, if $p$ is not geodesic at $t$.

DeFINITION 5.4. We will call a break-point $p(t)$ a turning point if the vectors $p_{-}^{\prime}(t), p_{+}^{\prime}(t)$ are linearly independent and a point of backtracking if $p_{-}^{\prime}(t)=-p_{+}^{\prime}(t)$.

Note that for $\eta \in V$, each chain in $W \eta$ can be extended to a chain which starts in $-\Delta$ and ends in $\Delta$. We leave the proof of the following lemma to the reader:

Lemma 5.5. Suppose that $\eta \in\left\{\varpi_{1}, \varpi_{2}\right\}$. Then every maximal chain in $W(\eta)$ has to be of the form:

$$
\eta_{0}, \eta_{1}=\tau_{1}\left(\eta_{0}\right), \eta_{2}=\tau_{2}\left(\eta_{1}\right), \ldots
$$

or

$$
\eta_{0}, \eta_{1}=\tau_{2}\left(\eta_{0}\right), \eta_{2}=\tau_{1}\left(\eta_{1}\right), \ldots
$$
of:

By combining these observations, we see that each chain in $W\left(\varpi_{1}\right)$ is a subchain

$$
\eta_{1}=-\varpi_{1}, \eta_{2}=\tau_{2}\left(\eta_{1}\right), \eta_{3}=\tau_{1}\left(\eta_{2}\right), \eta_{4}=\tau_{2}\left(\eta_{3}\right)=\varpi_{1}
$$

and each chain in $W\left(\varpi_{2}\right)$ is a subchain of:

$$
\eta_{1}=-\varpi_{2}, \eta_{2}=\tau_{1}\left(\eta_{1}\right), \eta_{3}=\tau_{2}\left(\eta_{2}\right), \eta_{4}=\tau_{1}\left(\eta_{3}\right)=\varpi_{2} .
$$

Accordingly, each $L S(R)$ path $p$ with length ${ }_{\Delta}(p) \in \mathbb{N} \varpi_{1}$ has the shape as in Figure 2 and each $L S(R)$ path with length $\Delta(p) \in \mathbb{N} \varpi_{2}$ has the shape as in Figure 3. (Some of the geodesic segments in $p$ described in these figures could have zero length.)

Lemma 5.6. Suppose $p$ is an LS path with respect to $2 R$ so that $p(0)$ is a special vertex of $\left(A, W_{a f f}\right)$, length $\Delta(p)=\mu \in \mathbb{Z}_{+} \cdot \varpi_{1}$ and all breaks of $p$ are at the vertices of $\left(A, W_{a f f}\right)$. Then:

1. $p$ has breaks only at the special vertices of $\left(A, W_{a f f}\right)$

2. $p_{1}$ is an $L S$ path with respect to $R$.

3. If $\mu \in 2 \mathbb{Z}_{+} \varpi_{1}$, then $p_{1}(0)-p_{1}(1) \in Q(R)$.

Proof. 1. Observe that our assumptions imply that the path $p_{1}$ has edges parallel to the coordinate axes and it is entirely contained in the 1-skeleton of the simplicial complex $\left(A, W_{a f f}\right)$. Let $v$ be a non-special vertex of $\left(A, W_{a f f}\right)$. Then $v$ is disjoint from all the edges of $\left(A, W_{a f f}\right)$ parallel to the coordinate axes. Therefore the path $p_{1}$ is disjoint from the set of nonspecial vertices.

2. Suppose that $v=p(t)$ is a break-point of $p$. Since $v$ is a special vertex of $\left(A, W_{a f f}\right)$, the chain condition in the definition of an LS path (with respect to $R$ ) at $v$ follows from the chain condition with respect to $2 R$. 


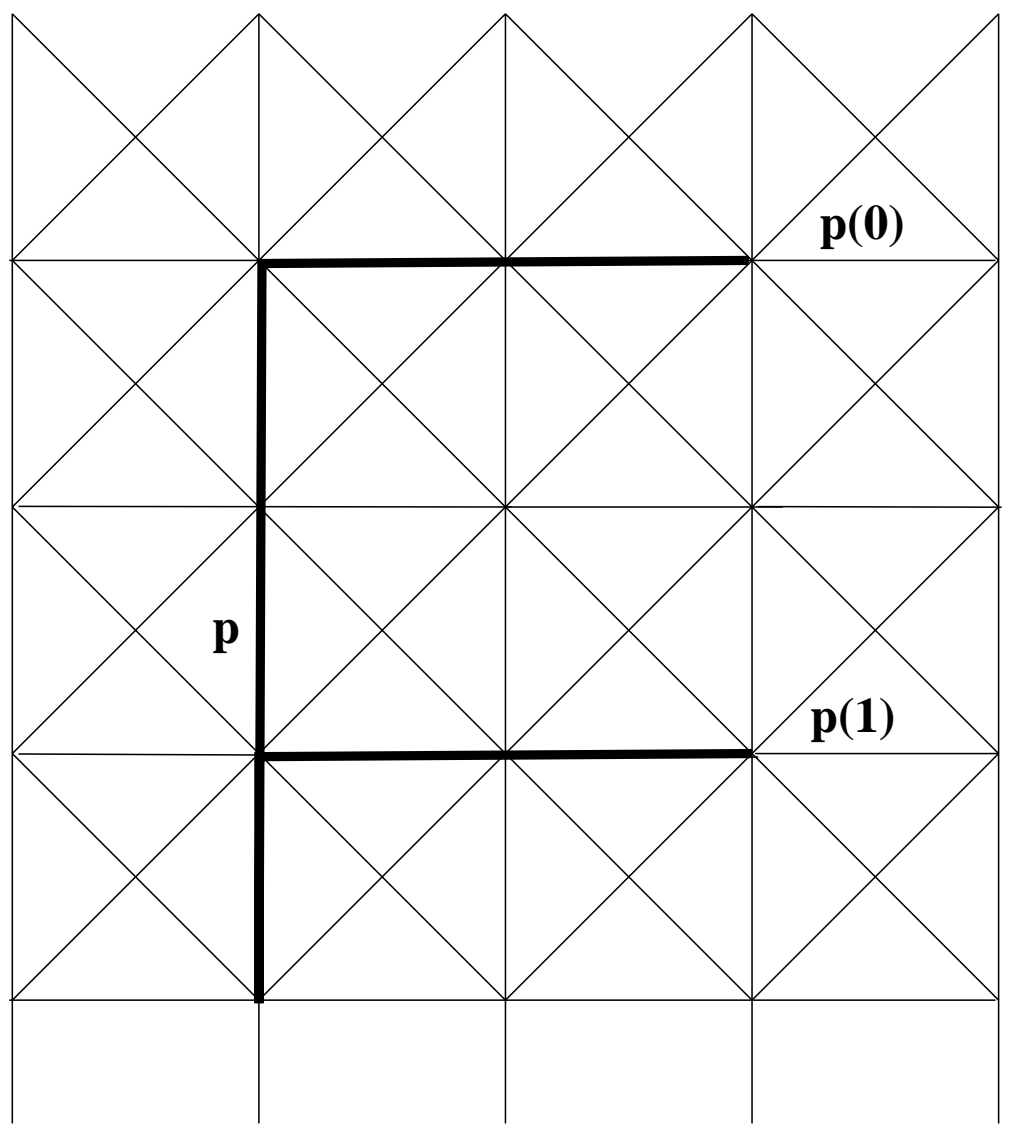

FIG. 2.

3. Since $p \in L S(R)$, it follows from Lemma 2.10 that

$$
p_{1}(0)-p_{1}(1)-\mu \in Q(R) .
$$

Since $\mu \in 2 \mathbb{Z}_{+} \cdot \varpi_{1} \subset Q(R)$, the last assertion of Lemma follows. $\square$

Corollary 5.7. Suppose that $p$ is an $L S(R)$-path with length $\Delta(p) \in \mathbb{N}_{\varpi_{1}}$. Then $p$ has breaks only at the special vertices of $\left(A, W_{a f f}\right)$.

Lemma 5.8. Suppose that $p=p_{1} * p_{2} \in L S_{1}(R)$. Then $p$ can have at most one break-point which is not a special vertex of $\left(A, W_{a f f}\right)$; such a point is a point of backtracking of the sub-path $p_{2}$.

Proof. By the previous lemma, the path $p_{1}$ can have breaks only at the special vertices of $\left(A, W_{a f f}\right)$. Consider the path $p_{2}$. Suppose that a break-point $p(t)$ is a turning point of $p$; let $\tau \in W_{\text {aff }}$ denote the reflection fixing $p(t)$ which sends $p_{-}^{\prime}(t)$ to $p_{+}^{\prime}(t)$. The vectors $p_{-}^{\prime}(t), p_{+}^{\prime}(t) \in T_{p(t)} A$ are tangent to the walls $H_{-}, H_{+}$of $\left(A, W_{a f f}\right)$ which are parallel to the lines $\{x=y\},\{x=-y\}$. The linear part of $\tau$ permutes $H_{-}, H_{+}$, hence $\tau$ is the reflection in a wall which is either vertical or horizontal. Therefore $p(t)$ is a special vertex of $\left(A, W_{a f f}\right)$. 


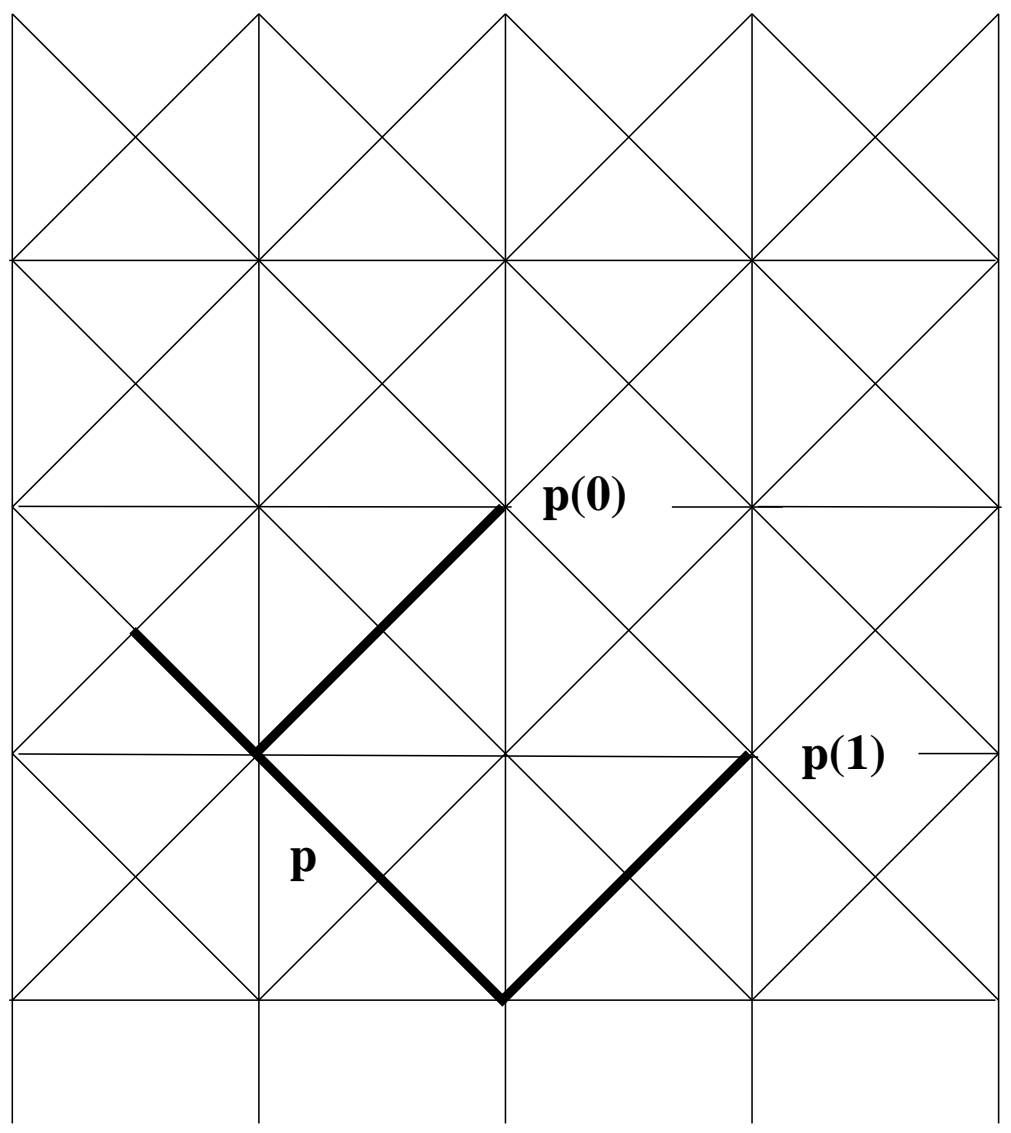

FIG. 3.

Hence, if a break-point $p$ is non-special, then the path $p$ has to backtrack at this point. On the other hand, our analysis of the shapes of LS paths shows that there could be at most one point where $p_{2}$ backtracks. $\square$

We now analyze the points of backtracking.

Lemma 5.9. Suppose that $p=p_{2}:[0,1] \rightarrow V$ belongs to $L S(R), \operatorname{length}_{\Delta}(p) \in$ $\mathbb{N} \varpi_{2}, p\left(t_{1}\right)$ is a backtracking point of $p$ which belongs to the wall $\{x=y\}$, and $p(0)$ or $p(1)$ belongs to $Q(R)$. Then $p\left(t_{1}\right)$ is a special vertex of $\left(A, W_{a f f}\right)$. line

Proof. Suppose that the break-point $p\left(t_{1}\right)=\left(x_{1}, x_{1}\right)$ is not special. Consider the

$$
\ell=\left\{x+y=2 x_{1}\right\} .
$$

Observe that the line $\ell$ contains no vertices of $Q(R)$ since $2 x_{1}$ is an odd number, while for each $(x, y) \in Q(R)$, the sum $x+y$ is even. Suppose that $p(0) \in Q(R)$. Then $p(0) \notin \ell$ and hence there exists a geodesic subsegment $\overline{p(0) p\left(t_{2}\right)}$ in $p$ which is orthogonal to $\ell$ so that $p\left(t_{2}\right)=\left(x_{2}, y_{2}\right) \in \ell$ is a turning point of $p$. However such segment clearly cannot contain points in $Q(R)$ since its points $(x, y)$ satisfy the equation

$$
x-y=x_{2}-y_{2} \notin 2 \mathbb{Z} .
$$


Contradiction.

As a corollary we obtain:

Corollary 5.10. Suppose that $\sigma=(\lambda, \mu, \nu) \in Q(R)^{3} \cap D_{3}$ and $\lambda+\mu+\nu \in 2 P(R)$. Then there exists a path $p \in L S_{1}\left(R^{\vee}\right)$ contained in $\Delta$ such that:

1. $p(0)=\lambda, p(1)=\nu$.

2. All break-points of $p$ are vertices of $\left(A, W_{a f f}\right)$.

Proof. Note that $Q(R)=2 P\left(R^{\vee}\right)$. We now apply the results established above to the root system $R^{\vee}$; note that this interchanges the roles of short and long fundamental weights, e.g. the wall $\{x=y\}$ contains the short fundamental weight of $R^{\vee}$.

Then, according to the saturation theorem 2.12, $\sigma \in \operatorname{Tens}\left(R^{\vee}\right)$. Moreover, there exists a path $p \subset \Delta$ connecting $\lambda$ to $\nu$ such that:

1. length $\Delta(p)=\left(\mu_{1}, \mu_{2}\right) \in 2 P\left(R^{\vee}\right) \times 2 P\left(R^{\vee}\right)$.

2. $\overline{p \in L S_{1}}\left(R^{\vee}\right)$ is a generalized LS path with respect to $R^{\vee}$.

Accordingly, the path $p$ is the concatenation $p=p_{1} * p_{2}$ of two $L S\left(R^{\vee}\right)$-paths $p_{i}:[0,1] \rightarrow V$. Consider the break-points of the path $p$. According to Corollary 5.7 , all break-points of $p_{2}$ are special vertices of $\left(A, W_{a f f}^{\vee}\right)$. All break-points of $p_{1}$ are special vertices of $\left(A, W_{a f f}^{\vee}\right)$ except possibly for a point $p_{2}\left(t_{1}\right)$ of backtracking which occurs on a wall of $\Delta$, where the path $p_{1}$ intersects $\partial \Delta$ orthogonally (see Theorem 2.12 and Lemma 5.8). On the other hand, the end-point $\lambda=p_{1}(0)$ of the path $p_{1}$ belongs to $2 P\left(R^{\vee}\right) \subset Q\left(R^{\vee}\right)$. Therefore, it follows from Lemma 5.9 (applied to the root system $R^{\vee}$ rather than $R$ ) that $p_{1}\left(t_{1}\right)$ has to be a special vertex of $\left(A, W_{a f f}^{\vee}\right)$.

Thus all break-points of $p$ occur in special vertices of $\left(A, W_{a f f}^{\vee}\right)$. Since $P\left(R^{\vee}\right)$ is the set of vertices of $\left(A, W_{a f f}\right)$, all break-points of $p$ are vertices of $\left(A, W_{a f f}\right)$.

5.2. Analysis of the $L S\left(R^{\vee}\right)$ paths with singular $\Delta$-length. In Corollary 5.10 we have established that for a large class of triples $(\lambda, \mu, \nu) \in D_{3}$, there exists an $L S_{1}\left(R^{\vee}\right)$ path $p$ with length $\Delta(p)=\mu$ connecting $\lambda$ to $\nu$, so that all break-points of $p$ are vertices of $\left(A, W_{a f f}\right)$.

We now analyze LS paths with respect to $R^{\vee}$ whose break-points are vertices of $\left(A, W_{a f f}\right)$. Such paths necessarily belong to $L S(2 R)$. Throughout this section we assume that $p:[0,1] \rightarrow V$ is a PL path in $\Delta$ so that:

- length $_{\Delta}(p)=\mu \in \mathbb{N} \varpi_{2}$.

- $\lambda:=p(0), \nu:=p(1) \in P(R), \lambda-\nu \in Q(R)$.

- All break-points of $p$ are vertices of $\left(A, W_{a f f}\right)$.

- $p \in L S\left(R^{\vee}\right)$.

Unlike the paths $p$ with $\operatorname{length}_{\Delta}(p) \in \mathbb{N} \varpi_{1}$, the above paths $p$ do not have to belong to $L S(R)$. However, after analyzing these paths, we show that in "most cases" they can be replaced with a new $L S(R)$ path $\hat{p}$ while keeping the same end-points and the $\Delta$-length.

If the path $p$ is not an LS path with respect to $R$ then it has a break-point which is not a special vertex of $\left(A, W_{a f f}\right)$.

Definition 5.11. Suppose that $v=p(t)$ is a break-point of a PL path $p$. We call the break-point $x$ illegal if it violates the axioms of an LS path (with respect to $R$ ).

Accordingly, we will refer to an illegal break-point which is a turning/backtracking point as an illegal turn/backtrack.

LEMMA 5.12. 1. Either $p$ has no illegal breaks or it has 2 illegal turns at $t_{1}<t_{2}$ or one illegal backtrack. 


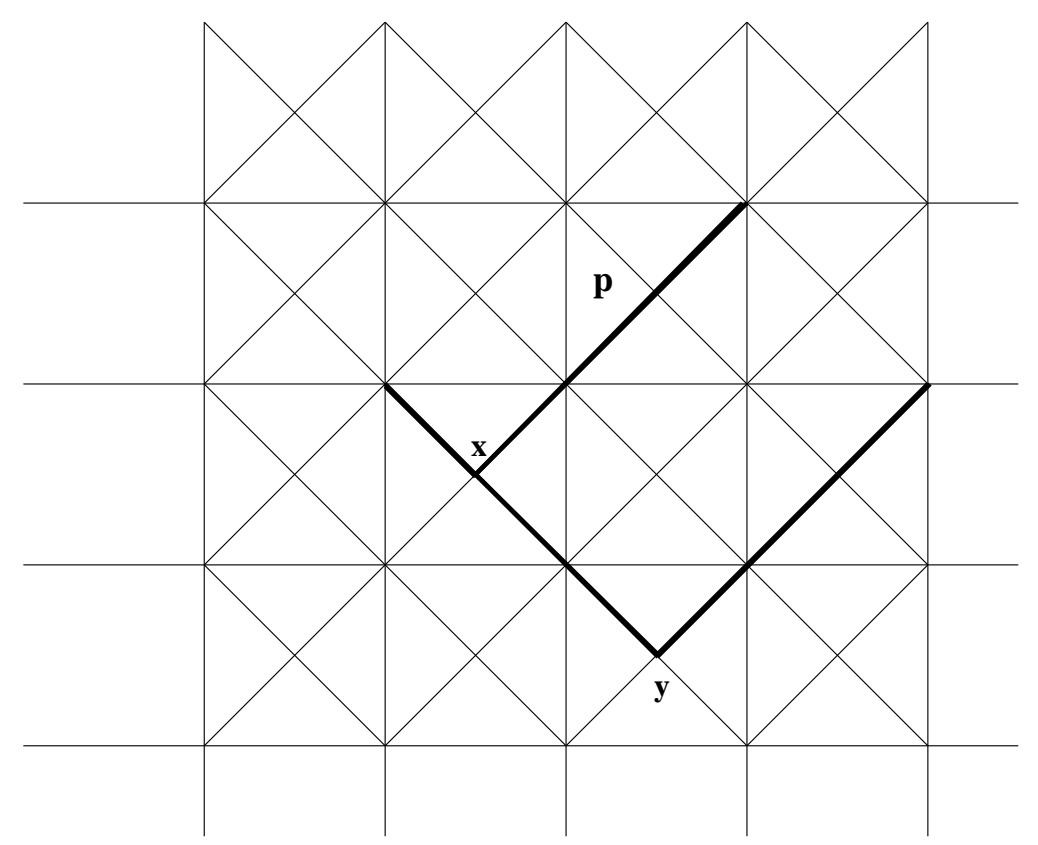

Fig. 4. Points $x, y$ are illegal breaks.

2. In the case of two illegal turns at $t_{1}, t_{2} \in[0,1]$, up to the reversal of orientation, the path $p$ has the shape described in the Figure 4.

Proof. We first consider the backtracks of $p$. If $p$ has a backtrack at $t$ and $p_{+}^{\prime}(t) \notin \Delta$ then

$$
w\left(p_{-}^{\prime}(t)\right)=p_{+}^{\prime}(t)
$$

where $w \in W_{a f f}^{\vee}$ is a reflection fixing the vertex $p(t)$ and whose linear part is $\tau_{1}$. Since $\tau_{1}$ is a simple reflection, it follows that the chain

$$
\left(p_{-}^{\prime}(t), p_{+}^{\prime}(t)\right)
$$

is necessarily maximal (see Lemma 3.15 in [13]) and therefore the backtrack at $t$ is legal. Moreover, the reflection $w$ also belongs to $W_{\text {aff }}$. Thus, if $p$ has an illegal backtrack at a point $t$ then $p_{-}^{\prime}(t) \in-\Delta, p_{+}^{\prime}(t) \in \Delta$. Therefore $p$ has no other breaks in this case and we are done. Hence we assume that $p$ has no illegal backtracks.

Let $t_{i} \in[0,1], i=1, \ldots, m$ denote the illegal turns of $p$. We set $t_{0}^{+}:=0, t_{m+1}^{-}:=1$. For each $t_{i}$ define $\left[t_{i}^{-}, t_{i}^{+}\right] \subset[0,1]$ to be the smallest subinterval containing $t_{i}$ such that $p\left(t_{i}^{-}\right), p\left(t_{i}^{+}\right)$are special vertices of $\left(A, W_{a f f}\right)$. Then the assumption that for each $i$ the point $p\left(t_{i}\right)$ is an illegal turn implies that

$$
p\left(t_{i}^{+}\right)-p\left(t_{i}^{-}\right) \notin Q(R) .
$$

On the other hand, the restriction of $p$ to each subinterval $\left[t_{i}^{+}, t_{i+1}^{-}\right]$is an LS path, therefore

$$
p\left(t_{i}^{+}\right)-p\left(t_{i+1}^{-}\right) \in Q(R)
$$


(see Lemma 2.10). Thus

$$
p(0)-p\left(t_{1}^{-}\right)+p\left(t_{1}^{+}\right)-p\left(t_{2}^{-}\right)+\ldots-p\left(t_{m}^{-}\right)+p\left(t_{m}^{+}\right)-p(1) \in Q(R)
$$

and since $p(0)-p(1) \in Q(R)$, the latter is equivalent to:

$$
\left(-p\left(t_{1}^{-}\right)+p\left(t_{1}^{+}\right)\right)+\ldots+\left(-p\left(t_{m}^{-}\right)+p\left(t_{m}^{+}\right)\right) \in Q(R) .
$$

Thus, since $Q(R)$ has index 2 in $P(R)$, it follows from (13) that $p$ has to have an even number of illegal turns. Since the length of the longest chain in $W \cdot \varpi_{2}$ is 4 , the number of illegal turns is $\leq 3$, hence it is either 0 or 2 . This proves the first assertion of Lemma.

Suppose that $p$ has illegal turns at $t_{1}$ and $t_{2}$, where $t_{1}<t_{2}$. Then, analogously to the case of an illegal backtrack,

$$
p_{-}^{\prime}\left(t_{1}\right) \in-\Delta, \quad p_{+}^{\prime}\left(t_{2}\right) \in \Delta .
$$

Therefore $\left.p\right|_{\left[0, t_{1}\right]},\left.p\right|_{\left[t_{2}, 1\right]}$ are geodesic paths. Moreover, since the length of the longest chain in $W \cdot \varpi_{2}$ is 4 , the path $p$ can have at most one (necessarily legal) break-point on the open interval $\left(t_{1}, t_{2}\right)$. Therefore the path $p$ has the shape as in Figure 4 .

Let $p$ be a path as above. Let $x_{1}=p\left(t_{1}\right), x_{2}=p\left(t_{2}\right)$ denote the first and the last illegal breaks of $p$ (possibly $t_{1}=t_{2}$ ). Then both breaks occur at nonspecial vertices. According to the above lemma, either $t_{1}=t_{2}$ and $p$ has an illegal backtrack at this point or both breaks are illegal turns. Let $t_{0}<t_{1}$ and $t_{3}>t_{2}$ be the largest and smallest values of $t$ such that $p(t) \in P(R)$. Our goal is to show that, with one exception, one can always modify the path $p$ on the interval $\left(t_{0}, t_{3}\right)$ (preserving its $\Delta$-length) so that the new path is an $L S(R)$-path.

Lemma 5.13. Suppose that $p(0) \notin \mathbb{N} \varpi_{2}$ and the image of $p$ is contained in $\Delta$. Then there exists a path $q:\left[t_{0}, t_{3}\right] \rightarrow V$ such that:

1. The concatenation

$$
\hat{p}:=\left.\left.p\right|_{\left[0, t_{0}\right]} * q * p\right|_{\left[t_{3}, 1\right]}
$$

is an $L S(R)$ path contained in $\Delta$.

2. $\operatorname{length}_{\Delta}(\hat{p})=\operatorname{length}_{\Delta}(p)$.

Proof. There are two cases to consider:

Case 1 . The path $p$ restricted to the open interval $\left(t_{1}, t_{2}\right)$ is not geodesic. In particular, $t_{1} \neq t_{2}$ and both illegal breaks are illegal turns. Then we use the modification described in Figure 5. It is clear that the new path $\hat{p}$ always satisfies the requirements of Lemma.

Case 2. The path $\left.p\right|_{\left(t_{1}, t_{2}\right)}$ is a (possibly constant) geodesic. Then we use the modification described in Figure 6 by introducing an extra break $\tau$ between $t_{1}$ and $t_{2}$.

It is clear that the new path $\hat{p}$ is an LS path, it has the same $\Delta$-length as $p$. However $\hat{p}$ is not necessarily contained in $\Delta$ : The point $q(\tau)$ could be outside of $\Delta$. This happens if and only if the point $p\left(t_{1}\right)$ lies on the wall $\{x=y\}$ of $\Delta$. In this case however $p(0) \in\{x=y\}$ as well which contradicts our hypothesis. $\square$

We now analyze in detail the exceptional case when $\lambda:=p(0)$ lies on the wall $\{x=y\}$, i.e. $\lambda$ belongs to $\mathbb{N}_{2}$. Then the path $p$ has exactly two turns or one backtrack and all breaks are illegal. 

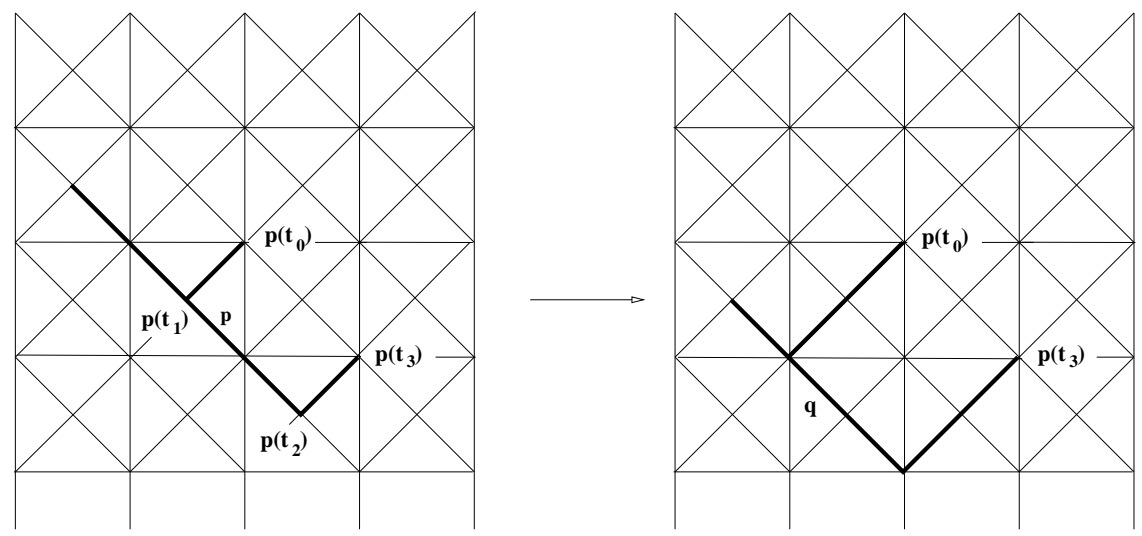

FIG. 5. Points $p\left(t_{1}\right), p\left(t_{2}\right)$ are illegal breaks of $p$.
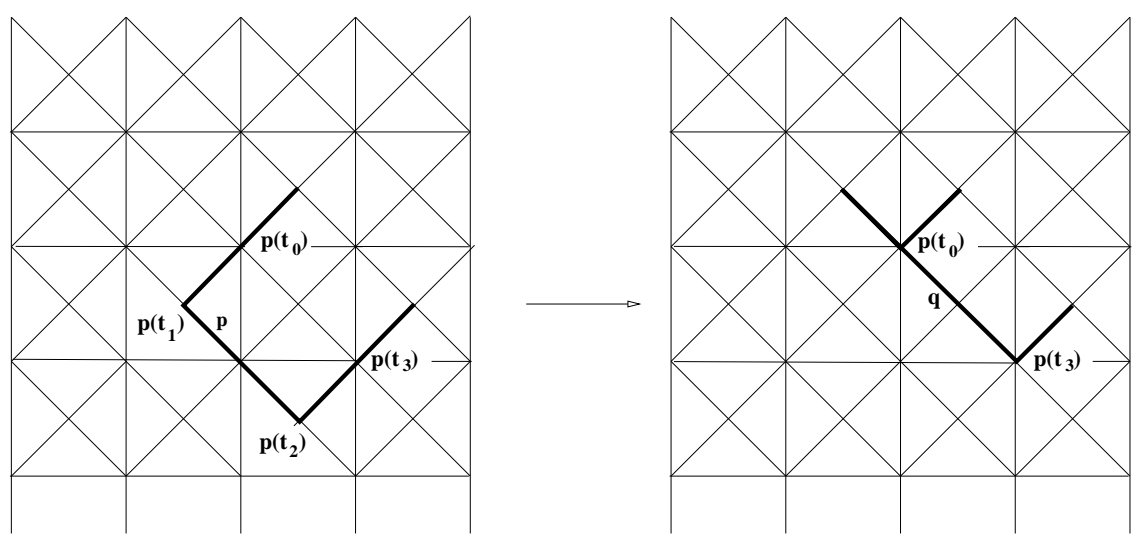

FIG. 6. Points $p\left(t_{1}\right), p\left(t_{2}\right)$ are illegal breaks of $p$.

LEMma 5.14. Under the above conditions we have:

$$
\lambda+\mu+\nu \notin 2 P(R) .
$$

Proof. Let $p$ have breaks at $t_{1} \leq t_{2}$. Set $u:=p\left(t_{1}\right), v:=p\left(t_{2}\right)$. Then

$$
\begin{gathered}
u=\lambda-\left(l+\frac{1}{2}\right) \varpi_{2}, v=\nu-\left(m+\frac{1}{2}\right) \varpi_{2}, \\
\mu=\tau_{2}\left(\left(l+\frac{1}{2}\right) \varpi_{2}+(v-u)+\left(m+\frac{1}{2}\right) \varpi_{2}\right),
\end{gathered}
$$

where $l, m \in \mathbb{Z}$ and $\tau_{2}(x, y)=(x,-y)$. Note that $\mu-\tau_{2}(\mu) \in 2 P(R)$. Therefore, modulo $2 P(R)$ we have:

$$
\lambda+\nu+\mu=\lambda+\nu+\left(l+\frac{1}{2}\right) \varpi_{2}+(v-u)+\left(m+\frac{1}{2}\right) \varpi_{2}=2 \nu+(2 l+1) \varpi_{2} .
$$

Hence $\lambda+\mu+\nu$ does not belong to $2 P(R)$. 


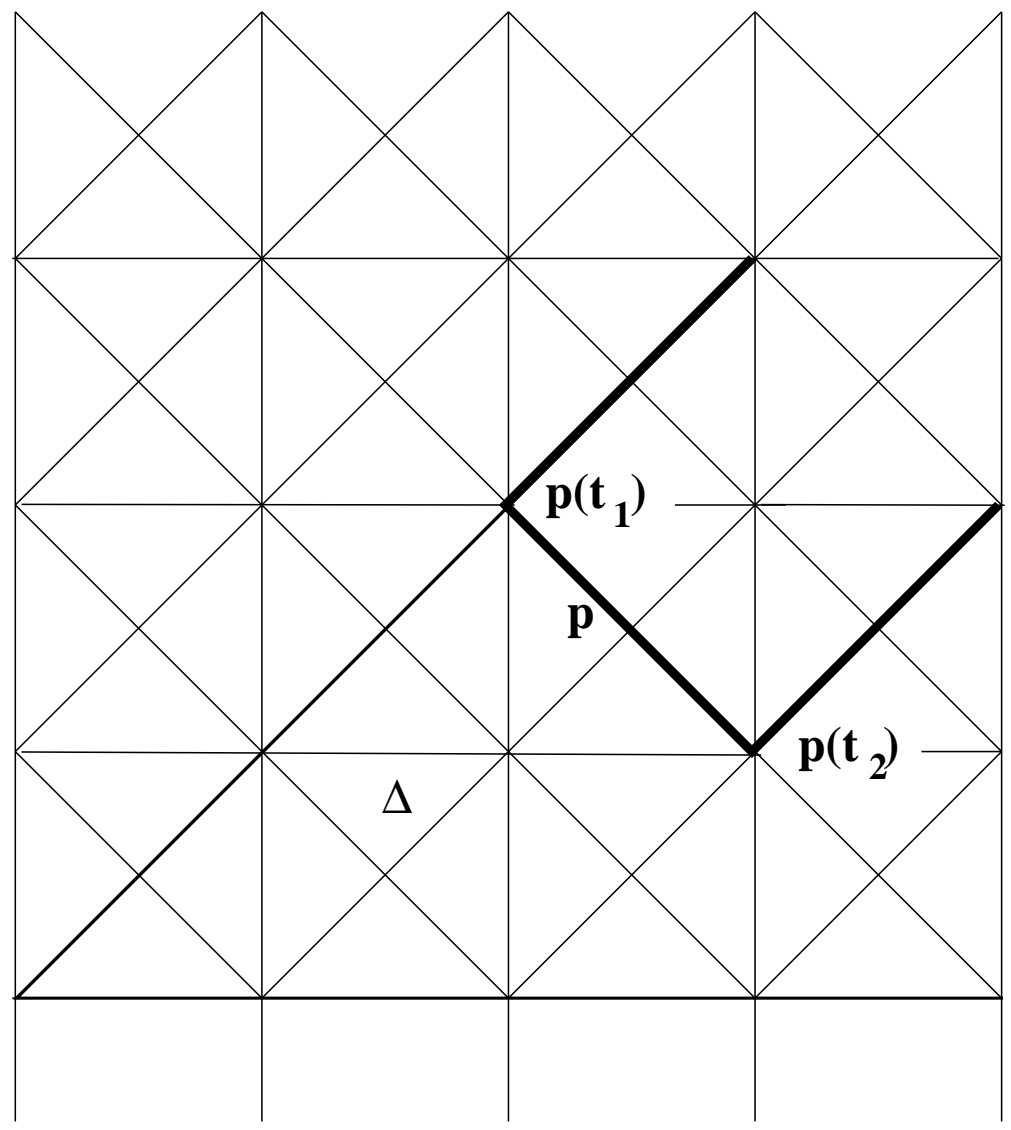

FIG. 7.

Lemma 5.15. If $\lambda=n \varpi_{2}$ and $(\lambda, \mu, \nu) \in$ Tens $(R)$ then $\lambda+\mu+\nu \in 2 P(R)$.

Proof. Suppose that $(\lambda, \mu, \nu) \in$ Tens $(R)$. Then there exists an LS path $q$ with $\operatorname{length}_{\Delta}(q)=\mu, q(0)=\lambda, q(1)=\nu$, so that $q$ is entirely contained in $\Delta$. This path is either geodesic or has one or two turns, or one backtrack. See Figure 7. We consider the "generic case" when $q$ has two turns at the points $u=q\left(t_{1}\right), v=q\left(t_{2}\right), t_{1}<t_{2}$. Then, analogously to the proof of the previous lemma,

$$
\begin{gathered}
\mu=(l+s+m) \varpi_{2}, \\
\nu=\lambda-l \varpi_{2}+s \tau_{2}\left(\varpi_{2}\right)+m \varpi_{2},
\end{gathered}
$$

where $u=\lambda-l \varpi_{2}, v=\nu-m \varpi_{2}$ and $l, s, m \in \mathbb{N}$. Since $\varpi_{2} \in P(R)$ and $\tau_{2}\left(\varpi_{2}\right)-\varpi_{2} \in$ $2 P(R)$, we obtain:

$$
\nu \equiv \lambda+l \varpi_{2}+s \varpi_{2}+m \varpi_{2}(\bmod 2 P(R))
$$

i.e.

$$
\nu \equiv \lambda+\mu \quad(\bmod 2 P(R))
$$


Therefore

$$
\lambda+\mu+\nu \equiv 2 \nu \equiv 0 \quad(\bmod 2 P(R)) .
$$

We summarize the above results in the following:

Proposition 5.16. Suppose that $\mu \in \mathbb{N}_{2}$ and $p$ is an LS path with respect to $2 R$ such that $p$ is contained in $\Delta, p(0)=\lambda, p(1)=\nu \in P(R), \operatorname{length}_{\Delta}(p)=\mu$ and all breaks of $p$ are at vertices of $\left(A, W_{\text {aff }}\right)$. Then:

1. $(\lambda, \mu, \nu) \notin$ Tens $(R)$ if and only if either $\lambda \in \mathbb{N}_{2}$ or $\nu \in \mathbb{N}_{2}$ and

$$
\lambda+\mu+\nu \notin 2 P(R) .
$$

2. Unless $p^{\prime}(0) \in-\Delta, p^{\prime}(1) \in \Delta$, the path $p$ is also an LS path with respect to the root system $R$.

3. Unless $\lambda$ or $\nu \in \mathbb{N} \varpi_{2}$, and $\lambda+\mu+\nu \notin 2 P(R)$, there exists a path $\hat{p}$ contained in $\Delta$ (of $\Delta$-length $\mu$ ) which is an $L S$ path with respect to the root system $R$, so that $\hat{p}(0)=p(0), \hat{p}(1)=p(1)$.

5.3. Analysis of $L S_{1}\left(R^{\vee}\right)$ paths. In the previous section we proved that for "most" singular $L S(2 R)$ paths $p_{i} \subset \Delta$ (with length $\Delta\left(p_{i}\right) \in \mathbb{N}^{\varpi_{i}}$ ), whose break-points are vertices of $\left(A, W_{a f f}\right)$, we can replace $p_{i}$ with a new path $\hat{p}_{i}$ which has the same $\Delta$-length, same end-points and is still contained in $\Delta$ (Proposition 5.16). The goal of this section is to prove a similar statement for paths $p=p_{1} * p_{2} \in L S_{1}(2 R)$. The naive idea would be to replace each $p_{i}$ with $\hat{p}_{i}$ using Proposition 5.16 and then take $\hat{p}:=\hat{p}_{1} * \hat{p}_{2}$. The are two issues however which have to be addressed:

(1) It might happen that the path $p_{2}$ is "exceptional" from the point of view of Proposition 5.17, i.e. $p_{2}(0) \in\{x=y\}$.

(2) We have to ensure that at the concatenation point between $\hat{p}_{1}$ and $\hat{p}_{2}$ the new path satisfies the axiom of an $L S_{1}(R)$ path.

It turns out that the issue (1) is trickier to handle: We cannot use Proposition 5.16 directly and are forced first to change the "concatenation point" (Figure 9) and move it away from the wall $\{x=y\}$.

The main result of this section is the following proposition:

Proposition 5.17. Suppose that $\sigma=(\lambda, \mu, \nu) \in P(R)^{3}$ is such that:

1. $\lambda, \nu \notin \mathbb{N} \varpi_{2}$.

2. There exists a path $p \in L S_{1}(2 R)$ from $\lambda$ to $\nu$, which is contained in $\Delta$, all whose break-points are vertices of $\left(A, W_{\text {aff }}\right)$ and so that

$$
\text { length }_{\Delta}(p)=\left(\mu_{1}, \mu_{2}\right), \mu=\mu_{1}+\mu_{2} \text {. }
$$

Then $\sigma \in \operatorname{Tens}(R)$.

Proof. We start by analyzing the path $p$. Our goal is to replace it with a new path which is in $L S_{1}(R)$ and which still satisfies condition 2 .

Set $\delta:=p_{1}(1)$. According to Lemma 5.6, the path $p_{1}$ is an LS path with respect to $R$. In particular, $\delta \in P(R)$. On the other hand, if $p_{2}^{\prime}(0)$ does not belong to $-\Delta$, then, according to the second part of Proposition 5.16, the path $p_{2}$ is an LS path with respect to $R$. Hence $p=p_{1} * p_{2}$ belongs to $L S_{1}(R)$, since the vertex $\delta$ is special and the generalized chain condition at this point (with respect to $R$ ) follows from the generalized chain condition at this point (with respect to $2 R$ ). 
We now consider the case $p_{2}^{\prime}(0) \in-\Delta$. Observe that, since $\delta$ is a special vertex, for each $\eta \in-\Delta$ and every $w \in W$,

$$
\eta \geq w(\eta)
$$

it follows that for every $L S(R)$-path $q_{2}$, the concatenation

$$
p_{1} * q_{2}
$$

belongs to $L S_{1}(R)$.

Case 1. $p_{1}(1) \notin\{x=y\}$. Then, according to Part 3 of Proposition 5.16, there exists an $L S(R)$-path $q_{2}$ (entirely contained in $\Delta$ ) starting at $\delta$, ending at $\nu$, with length $_{\Delta}\left(q_{2}\right)=\mu_{2}$. Hence the concatenation $\hat{p}:=p_{1} * q_{2}$ is a generalized LS path with respect to the root system $R, \hat{p}$ is contained in $\Delta$, and therefore $(\lambda, \mu, \nu) \in \operatorname{Tens}(R)$.

Case 2. $p_{1}(1) \in\{x=y\}$. Since $p_{2}^{\prime}(0) \in-\Delta$, then $p_{1}^{\prime}(1) \in \mathbb{R}_{-} \times \mathbb{R}_{-}$. Since $\delta=p_{1}(1)$ belongs to the wall $\{x=y\}$ and $p_{1}$ is contained in $\Delta$, it follows that $p_{1}^{\prime}(1) \in-\Delta$. Hence $p_{1}$ is a geodesic path and the entire path $p$ has the shape as in Figure 8 .

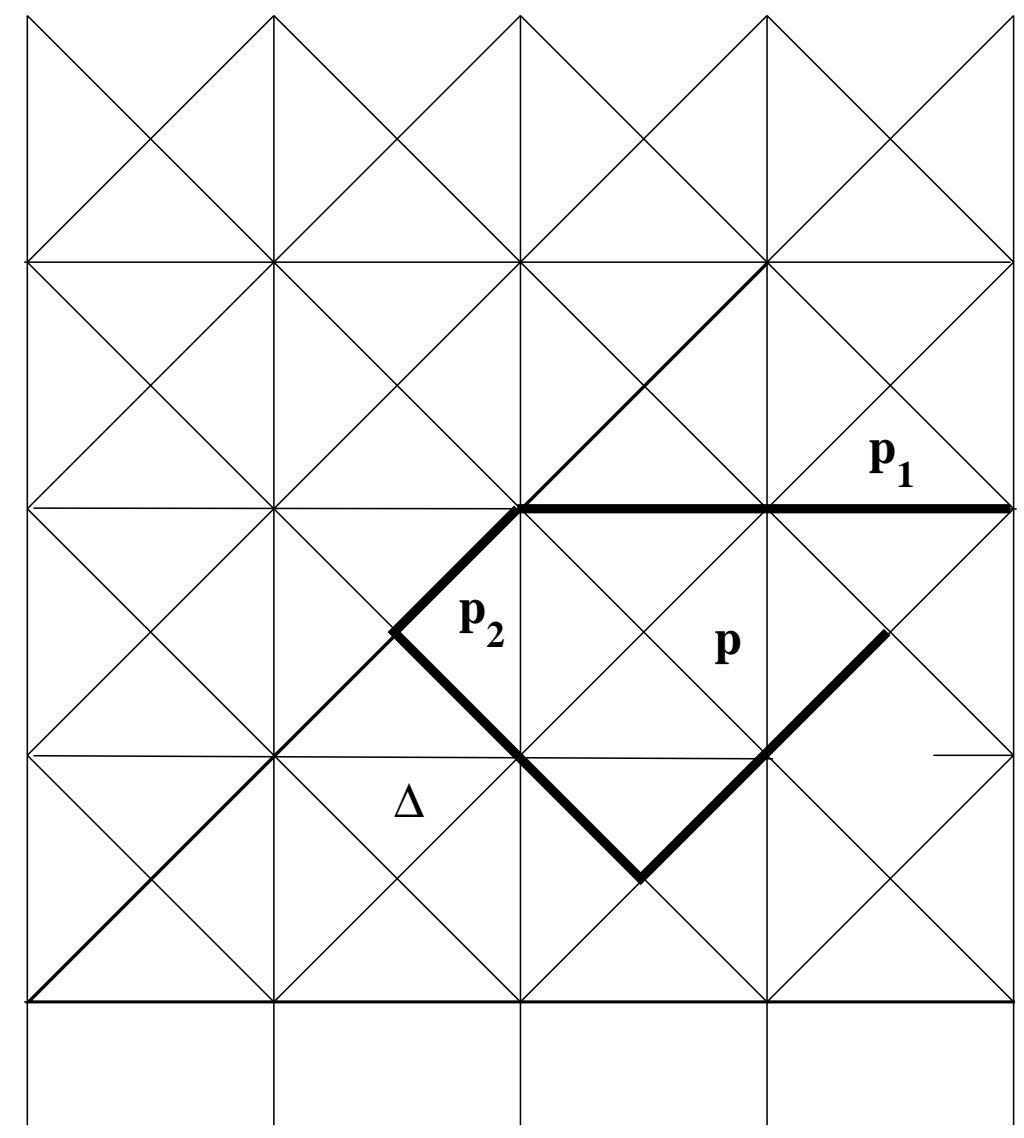

FIG. 8.

We let $t_{1} \in[0,1]$ be such that $\delta=p\left(t_{1}\right)$ is the concatenation point, let $t_{0}<t_{1}$ be the maximal value of $t$ such that $p(t)$ is a special vertex. Let $t_{2}>t_{1}$ be the 
first value of $t$ where $p(t)$ is not geodesic, $t_{3}>t_{2}$ be the first value of $t$ such that $p\left(t_{2}\right)$ is a (special) vertex. We now replace the restriction $\left.p\right|_{\left[t_{0}, t_{3}\right]}$ with the new path $\tilde{p}:\left[t_{0}, t_{3}\right] \rightarrow V$ described in Figure 9. Observe that $p\left(t_{3}\right) \notin \partial \Delta$, for otherwise the path $p_{2}$ has exactly one illegal turn which contradicts Lemma 5.12. Therefore the path $\tilde{p}$ is contained in $\Delta$.

Moreover, $\tilde{p}\left(t_{0}\right)=p\left(t_{0}\right), \tilde{p}\left(t_{3}\right)=p\left(t_{3}\right)$,

$$
\underline{\text { length }} \Delta(\tilde{p})=\underline{\text { length }} \Delta\left(\left.p\right|_{\left[t_{0}, t_{3}\right]}\right) .
$$

Thus we define the path

$$
q:=\left.\left.p\right|_{\left[0, t_{0}\right]} * \tilde{p}\left[t_{0}, t_{3}\right] * p\right|_{\left[t_{3}, 1\right]} .
$$

Note that the new path has a (legal) turn at the point $\tilde{p}\left(t_{3}\right)$ and in addition, two (illegal) simple turns. Therefore $q=q_{1} * q_{2}$ is still not a generalized LS path. However it has the property that its concatenation point $\tilde{\delta}=q\left(t_{1}\right)$ is not on the wall $\{x=y\}$. Thus we have reduced the argument to Case 1 and hence $\sigma \in \operatorname{Tens}(R)$.
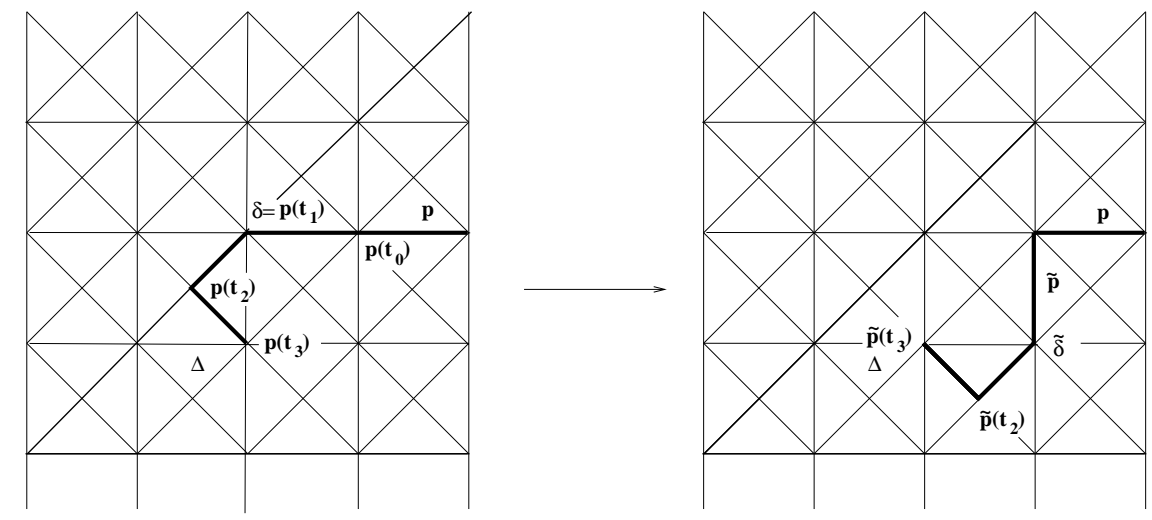

FIG. 9.

This concludes the proof of Proposition 5.17.

Corollary 5.18. Suppose that $\sigma=(\lambda, \mu, \nu) \in Q(R)^{3} \cap D_{3}$ and $\lambda+\mu+\nu \in 2 P(R)$. Then $\sigma \in \operatorname{Tens}(R)$.

Proof. If all three vectors $\lambda, \mu, \nu$ do not belong to $\mathbb{N} \varpi_{2}$ then the assertion follows immediately from the combination of Corollary 5.10 and Proposition 5.17. If one of these vectors belongs to $\mathbb{N}_{2}$ (by permuting $\lambda, \mu, \nu$ we can assume that this vector is $\mu$ ), then we use Corollary 5.10 and Proposition 5.16.

5.4. Proof of Theorem 5.3. Our final goal is to eliminate the assumption that $\lambda, \mu, \nu \in Q(R)$ in Corollary 5.18. Recall that $(\lambda, \mu, \nu) \in D_{3} \cap(P(R))^{3}, \lambda+\mu+\nu \in Q(R)$ and either at most one of the vectors $\lambda, \mu, \nu$ belongs to $\mathbb{N}_{2}$ or $\lambda+\mu+\nu \in 2 P(R)$. If one of the vectors $\lambda, \mu, \nu$ belongs to $\mathbb{N}_{2}$, we can assume (by relabelling) that this vector is $\mu$. (Note that $\varpi_{2} \in Q(R)$ which makes this relabelling consistent with our convention 5.1.)

Observe that $\lambda, \mu, \nu \in P(R)=Q\left(R^{\vee}\right), \lambda+\mu+\nu \in Q(R)=2 P\left(R^{\vee}\right)$. Therefore, since the root systems $B_{2}$ and $C_{2}$ are isomorphic, we can apply Corollary 5.18 to 
the triple $(\lambda, \mu, \nu)$ with respect to the coroot system $R^{\vee}$ and conclude that the triple $(\lambda, \mu, \nu)$ belongs to Tens $\left(R^{\vee}\right)$.

Hence there exists a generalized LS path $p$ in $\Delta$ with respect to the root system $R^{\vee}$, connecting $\lambda$ to $\mu$ and having

$$
\underline{\text { length }}_{\Delta}(p)=\left(\mu_{1}, \mu_{2}\right) \text {. }
$$

This path is a concatenation $p=p_{1} * p_{2}$ of $L S\left(R^{\vee}\right)$ paths $p_{1}, p_{2}$, the edges of the path $p_{1}$ are parallel to the $x$ and $y$ axes.

REMARK 5.19. Strictly speaking, since in the root system $R^{\vee}$ the long and the short roots are reversed, according to our notation 5.2, we would have to use the concatenation $p_{2} * p_{1}$ rather than $p_{1} * p_{2}$. However reversing the roles of $\lambda$ and $\nu$ eliminates this problem.

LEMma 5.20. The breaks of $p$ occur only at special vertices of the Coxeter complex $\left(A, W_{a f f}^{\vee}\right)$.

Proof. Observe that our analysis of generalized LS paths (see Lemma 5.8 applied to the root system $R^{\vee}$ ) shows that the break-points of $p$ occur only at special vertices of $\left(A, W_{a f f}^{\vee}\right)$ with the sole exception of a single break of the sub-path $p_{1}$ which can occur at a nonspecial vertex $p\left(t_{1}\right)$, and where $p$ backtracks and has germ parallel to the $y$ axis.

We claim that this is impossible. Let $\left[t_{0}, t_{2}\right]$ be the smallest interval containing $t_{1}$ such that $p\left(t_{0}\right)=p\left(t_{1}\right) \in P\left(R^{\vee}\right)$. We then replace the path $p$ with the path $\tilde{p}$ by eliminating this backtracking:

$$
\tilde{p}=\left.\left.p\right|_{\left[0, t_{0}\right]} * p\right|_{\left[t_{1}, 1\right]} .
$$

Then

$$
\tilde{\mu}:=\operatorname{length}_{\Delta}(\tilde{p})=\operatorname{length}_{\Delta}(p)-\varpi_{1} .
$$

It is clear that the new path $\tilde{p}$ is a generalized LS path with respect to the root system $R^{\vee}$ and moreover its breaks occur only at the special vertices of $\left(A, W_{a f f}^{\vee}\right)$, i.e. at vertices of $\left(A, W_{a f f}\right)$. We are now in position to apply Proposition 5.17 (with respect to the root system $R$ ).

1. Either $(\lambda, \tilde{\mu}, \nu) \in \operatorname{Tens}(R)$,

2. Or two of the vectors $\lambda, \mu, \nu$ belong to $\mathbb{N} \varpi_{2}$.

In Case 1, since $(\lambda, \tilde{\mu}, \nu) \in$ Tens $(R)$, it follows that

$$
\lambda+\mu+\nu-\varpi_{1}=\lambda+\tilde{\mu}+\nu \in Q(R) .
$$

However $\lambda+\mu+\nu \in Q(R)$ (by the assumption in Theorem 5.3) and $\varpi_{1} \notin Q(R)$. Contradiction.

In Case 2, as it was observed in the beginning of this section, we can assume that $\mu \in \mathbb{N} \varpi_{2}$. Then the path $p$ cannot contain a subsegment parallel to the $y$-axis, i.e. the above backtracking in the path $p$ is impossible.

Corollary 5.21. There exists a path $p$ in $\Delta$, connecting $\lambda$ to $\nu$, which is a generalized LS path with respect to $R^{\vee}$ all whose breaks are at vertices of $\left(A, W_{\text {aff }}\right)$ and such that length ${ }_{\Delta}(p)=\left(\mu_{1}, \mu_{2}\right)$. 
We now can finish the proof of Theorem 5.3. Consider a path $p$ in $\Delta$ as in Corollary 5.21. The breaks in this path occur only in vertices of $\left(A, W_{a f f}\right)$. Suppose that at most one of the vectors $\lambda, \mu, \nu$ belongs to $\mathbb{N} \varpi_{2}$. Then, according to Proposition $5.17, \sigma \in \operatorname{Tens}(R)$.

Consider the exceptional case, say, $\lambda, \mu \in \mathbb{N}_{2}$. Then, according to the hypothesis of Theorem 5.3, $\lambda+\mu+\nu \in 2 P(R)$. Therefore we can apply Proposition 5.16 and $\sigma \in \operatorname{Tens}(R)$.

Below we express $\operatorname{Tens}(R)$ as a union of elementary sets, where $G=S p(4, \mathbb{C})$. In what follows, $\mathbb{Z}_{+}=\{0,1,2, \ldots\}$. Let

$$
\begin{gathered}
E_{1}=\left\{(\lambda, \mu, \nu) \in L^{3}: \lambda+\mu+\nu \in 2 P(R), \lambda \in \mathbb{Z}_{+} \varpi_{2}, \mu \in \mathbb{Z}_{+} \varpi_{2}\right\} \cap \mathcal{P}(G), \\
E_{2}=\left\{(\lambda, \mu, \nu) \in L^{3}: \lambda+\mu+\nu \in 2 P(R), \lambda \in \mathbb{Z}_{+} \varpi_{2}, \nu \in \mathbb{Z}_{+} \varpi_{2}\right\} \cap \mathcal{P}(G), \\
E_{3}=\left\{(\lambda, \mu, \nu) \in L^{3}: \lambda+\mu+\nu \in 2 P(R), \nu \in \mathbb{Z}_{+} \varpi_{2}, \mu \in \mathbb{Z}_{+} \varpi_{2}\right\} \cap \mathcal{P}(G), \\
E_{1}^{\prime}=\left\{(\lambda, \mu, \nu) \in \Lambda: \lambda \notin \mathbb{Z}_{+} \varpi_{2}, \mu \notin \mathbb{Z}_{+} \varpi_{2}\right\} \cap \mathcal{P}(G), \\
E_{2}^{\prime}=\left\{(\lambda, \mu, \nu) \in \Lambda: \lambda \notin \mathbb{Z}_{+} \varpi_{2}, \nu \notin \mathbb{Z}_{+} \varpi_{2}\right\} \cap \mathcal{P}(G), \\
E_{3}^{\prime}=\left\{(\lambda, \mu, \nu) \in \Lambda: \mu \notin \mathbb{Z}_{+} \varpi_{2}, \nu \notin \mathbb{Z}_{+} \varpi_{2}\right\} \cap \mathcal{P}(G) .
\end{gathered}
$$

Then

$$
\text { Tens }=\bigcup_{i=1}^{3} E_{i} \cup \bigcup_{i=1}^{3} E_{i}^{\prime} .
$$

6. Computation of $\operatorname{Tens}\left(G_{2}\right)$. Let $R$ be the root system $G_{2}$, and let $L:=P(R)$ denote the weight lattice. We let $\varpi_{1}, \varpi_{2}$ denote the fundamental weights of $R$ so that $\varpi_{2}$ is the longer weight. Let $H_{i}$ denote the walls $\mathbb{R} \varpi_{i}, i=1,2$. We will use the coordinates $[x, y]$ for vectors $\lambda=x \varpi_{1}+y \varpi_{2}$ in $V=P(R) \otimes \mathbb{R}$, so that the chamber $\Delta$ is given by the inequalities $x \geq 0, y \geq 0$. Let $G$ be the complex simple Lie group with the root system $R$ and maximal compact subgroup $K$. Recall that $\mathcal{P}(G)=D_{3}(G / K) \subset \Delta^{3}$ denotes the convex cone given by the stability and chamber inequalities. Note that the permutation group on 3 elements $S_{3}$ acts on $\mathcal{P}(G)$ (by permuting $\left.\lambda_{1}, \lambda_{2}, \lambda_{3}\right)$ and this action preserves $\operatorname{Tens}(G)$.

The following theorem gives a complete description of the semigroup Tens $(G)$.

TheOREM 6.1. Suppose that $\sigma=\left(\lambda_{1}, \lambda_{2}, \lambda_{3}\right) \in \mathcal{P}(G) \cap L^{3}$. Then:

1. If at most one of the vectors $\lambda_{i}$ is a multiple of $\varpi_{2}$ then $\sigma$ belongs to Tens $(G)$.

2. Suppose that $\lambda_{1}=y_{1} \varpi_{2}, \lambda_{2}=y_{2} \varpi_{2}$. Then $\sigma \notin T e n s(G)$ if and only if $\sigma$ belongs to the union $\mathcal{E}_{1} \cup \mathcal{E}_{2} \cup \mathcal{E}_{3}$ of the following "exceptional" elementary sets:

$$
\begin{gathered}
\mathcal{E}_{1}=\left\{\left(\left[\begin{array}{c}
0 \\
y_{1}
\end{array}\right],\left[\begin{array}{c}
0 \\
y_{2}
\end{array}\right],\left[\begin{array}{c}
1 \\
y_{3}
\end{array}\right]\right): y_{1}, y_{2}, y_{3} \in \mathbb{Z}_{+}\right\}, \\
\mathcal{E}_{2}=\left\{\left(\left[\begin{array}{c}
0 \\
1+n+m
\end{array}\right],\left[\begin{array}{c}
0 \\
1+n+2 m
\end{array}\right],\left[\begin{array}{c}
1+3 m \\
0
\end{array}\right]\right): n, m \in \mathbb{Z}_{+}\right\},
\end{gathered}
$$




$$
\mathcal{E}_{3}=\left\{\left(\left[\begin{array}{c}
0 \\
1+n+m
\end{array}\right],\left[\begin{array}{c}
0 \\
1+m
\end{array}\right],\left[\begin{array}{c}
1+3 m \\
1+n
\end{array}\right]\right): n, m \in \mathbb{Z}_{+}\right\} .
$$

Note that the sets $\mathcal{E}_{2}, \mathcal{E}_{3}$ can be also described as follows. Let $\phi_{i}(\sigma)=\phi_{i}\left(\lambda_{1}, \lambda_{2}, \lambda_{3}\right)$, $i=0,1,2$ be given by

$$
\begin{gathered}
\phi_{0}(\sigma)=\left(2 x_{1}-x_{2}+x_{3}\right)+3\left(y_{1}-y_{2}+y_{3}\right), \\
\phi_{1}(\sigma)=\left(x_{1}+x_{2}-x_{3}\right)+\left(y_{1}+2 y_{2}-y_{3}\right), \\
\phi_{2}(\sigma)=\left(x_{1}+x_{2}-x_{3}\right)+3\left(y_{1}+y_{2}-y_{3}\right) .
\end{gathered}
$$

The inequalities $\phi_{i}(\sigma) \geq 0, i=0,1,2$, appear in the system of stability inequalities defining $\mathcal{P}(G)$ (see $[10]$ ). Then

$$
\begin{gathered}
\mathcal{E}_{2}=\left\{\sigma=\left(\lambda_{1}, \lambda_{2}, \lambda_{3}\right): \lambda_{1}, \lambda_{2} \in \mathbb{Z} \varpi_{2}, \lambda_{3} \in \mathbb{Z} \varpi_{1}, \phi_{0}(\sigma)=1\right\}, \\
\mathcal{E}_{3}=\left\{\sigma=\left(\lambda_{1}, \lambda_{2}, \lambda_{3}\right): \lambda_{1}, \lambda_{2} \in \mathbb{Z} \varpi_{2}, \phi_{i}(\sigma)=1, i=1,2\right\} .
\end{gathered}
$$

Thus $\mathcal{E}_{2}, \mathcal{E}_{3}$ are sets of lattice points (i.e. elements of $L$ ) in translates of strata of the boundary of the cone $\mathcal{P}(G)$.

We get the following corollaries of the above theorem:

Corollary 6.2. If $\sigma \in \mathcal{P}(G) \cap L^{3}$ is a nonsingular triple then $\sigma \in \operatorname{Tens}(G)$.

Corollary 6.3. In the decomposition of Tens $(G)$ as the union of elementary sets, the elementary sets are given by inequalities only and there are no congruence conditions.

Proof. (of Theorem 6.1). The proof that each triple $\sigma$ which is not in $S_{3} \cdot\left(\mathcal{E}_{1} \cup\right.$ $\left.\mathcal{E}_{2} \cup \mathcal{E}_{3}\right)$ belongs to Tens $(G)$ is a, rather uninteresting, computation. The proof that $\left(\mathcal{E}_{1} \cup \mathcal{E}_{2} \cup \mathcal{E}_{3}\right) \cap$ Tens $(G)=\emptyset$ is based on analysis of LS paths (and generalized LS paths) with respect to the root system $G_{2}$.

Notation 6.4. We define the following elements of L (see Figure 10):

$$
\begin{gathered}
\alpha_{0}:=\varpi_{2}, \alpha_{1}:=3 \varpi_{1}-\varpi_{2}, \alpha_{2}:=\alpha_{0}-\alpha_{1} . \\
\beta_{0}:=\varpi_{1}, \beta_{1}:=\varpi_{2}-\beta_{0}, \beta_{2}:=\beta_{0}-\beta_{1} .
\end{gathered}
$$

The following observation will be very useful for the proofs of Propositions 6.12, 6.17 below:

ObSeRvation 6.5. Let $\left(A, W_{a f f}\right)$ be the affine Coxeter complex for the root system $R=G_{2}$ and $W$ be the finite Weyl group of $R$. Suppose that $W^{\prime}$ is the stabilizer of a vertex $v$ in $\left(A, W_{\text {aff }}\right)$; we identify $W^{\prime}$ with a subgroup of $W$. Let $\eta_{0} \geq \eta_{1} \geq \ldots \geq$ $\eta_{m}=\alpha_{0}$ is a $W^{\prime}$-chain which is maximal as a $W$-chain. Then $W^{\prime}=W$ and $v$ is a special vertex. 


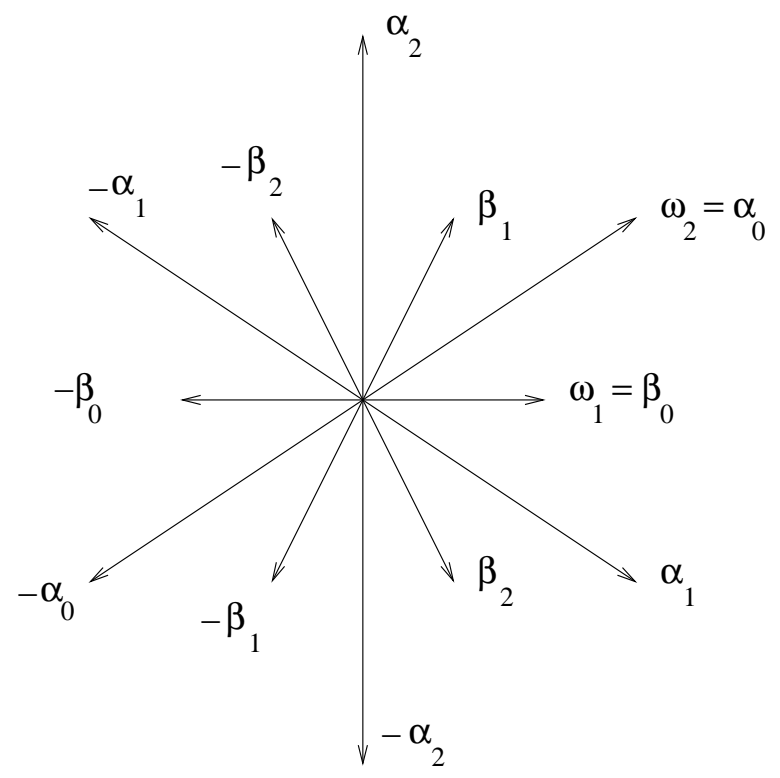

FIG. 10 .

Definition 6.6. Let $p:[0,1] \rightarrow V$ be a Hecke path. We say that $p$ is modeled on a chain $\eta_{0} \geq \ldots \geq \eta_{m}$ if the following holds:

Let $t_{0}=0<\ldots<t_{m}<1$, where $t_{1}, \ldots, t_{m}$ denote the break-points of $p$. Since $p$ is a Hecke path, the sequence $\zeta_{0}=p^{\prime}\left(t_{0}\right), \zeta_{1}=p_{+}^{\prime}\left(t_{1}\right), \ldots, \zeta_{m}=p_{+}^{\prime}\left(t_{m}\right)$ is a chain. We then require each $\zeta_{i}$ to be a multiple of $\eta_{i}, i=0, \ldots, m$.

We let $w=(23), u=(13), v=(312)$ denote permutations in $S_{3}$.

Proposition 6.7. Suppose that $\sigma=\left(\lambda_{1}, \lambda_{2}, \lambda_{3}\right) \in \mathcal{P}(G) \cap L^{3}$, is such that at most one vector $\lambda_{i}$ is a multiple of $\varpi_{2}$. Then $\sigma \in$ Tens $(G)$.

Proof. Recall that in [12] we have computed the semigroup generators for $\mathcal{P}(G) \cap$ $L^{3}$. These are the following triples $\delta_{i}, \epsilon_{j}, i=1, \ldots, 9, j=1,2$, and their images under the $S_{3}$-action:

$$
\begin{aligned}
& \delta_{1}:=\left(\left[\begin{array}{l}
1 \\
0
\end{array}\right],\left[\begin{array}{l}
1 \\
0
\end{array}\right],\left[\begin{array}{l}
0 \\
0
\end{array}\right]\right), \quad \delta_{2}:=\left(\left[\begin{array}{l}
0 \\
1
\end{array}\right],\left[\begin{array}{l}
0 \\
1
\end{array}\right],\left[\begin{array}{l}
0 \\
0
\end{array}\right]\right), \\
& \delta_{3}:=\left(\left[\begin{array}{l}
1 \\
0
\end{array}\right],\left[\begin{array}{l}
1 \\
0
\end{array}\right],\left[\begin{array}{l}
1 \\
0
\end{array}\right]\right), \quad \delta_{4}:=\left(\left[\begin{array}{l}
0 \\
1
\end{array}\right],\left[\begin{array}{l}
0 \\
1
\end{array}\right],\left[\begin{array}{l}
0 \\
1
\end{array}\right]\right), \\
& \delta_{5}:=\left(\left[\begin{array}{l}
0 \\
1
\end{array}\right],\left[\begin{array}{l}
0 \\
1
\end{array}\right],\left[\begin{array}{l}
3 \\
0
\end{array}\right]\right), \quad \delta_{6}:=\left(\left[\begin{array}{l}
0 \\
1
\end{array}\right],\left[\begin{array}{l}
0 \\
2
\end{array}\right],\left[\begin{array}{l}
3 \\
0
\end{array}\right]\right), \\
& \delta_{7}:=\left(\left[\begin{array}{l}
0 \\
1
\end{array}\right],\left[\begin{array}{l}
1 \\
0
\end{array}\right],\left[\begin{array}{l}
1 \\
0
\end{array}\right]\right), \quad \delta_{8}:=\left(\left[\begin{array}{l}
0 \\
1
\end{array}\right],\left[\begin{array}{l}
1 \\
0
\end{array}\right],\left[\begin{array}{l}
2 \\
0
\end{array}\right]\right),
\end{aligned}
$$




$$
\begin{gathered}
\delta_{9}:=\left(\left[\begin{array}{l}
0 \\
1
\end{array}\right],\left[\begin{array}{l}
0 \\
1
\end{array}\right],\left[\begin{array}{l}
2 \\
0
\end{array}\right]\right), \\
\epsilon_{1}:=\left(\left[\begin{array}{l}
0 \\
1
\end{array}\right],\left[\begin{array}{l}
0 \\
1
\end{array}\right],\left[\begin{array}{l}
1 \\
0
\end{array}\right]\right), \epsilon_{2}:=\left(\left[\begin{array}{l}
0 \\
1
\end{array}\right],\left[\begin{array}{l}
0 \\
1
\end{array}\right],\left[\begin{array}{l}
1 \\
1
\end{array}\right]\right) .
\end{gathered}
$$

It was observed in [12] that only $\epsilon_{1}, \epsilon_{2}$ are not in Tens $(G)$. Moreover,

$$
\epsilon_{1}+\epsilon_{2}=\delta_{4}+\delta_{9} \in \operatorname{Tens}(G), \quad \epsilon_{1}+u\left(\epsilon_{2}\right)=\delta_{4}+u\left(\delta_{1}\right)+u\left(\delta_{2}\right) \in \operatorname{Tens}(G)
$$

and for each natural number $n \geq 2, n \epsilon_{i}$ belongs to Tens $(G), i=1,2$.

Therefore, if $\sigma=\left(\lambda_{1}, \lambda_{2}, \lambda_{3}\right)$ is a combination of the semigroup generators which is not in $\operatorname{Tens}(G)$, then it has the form

$$
\epsilon_{i}+\sum_{g \in S_{3}} \sum_{j=1}^{9} n_{g j} g\left(\delta_{j}\right),
$$

where $n_{g j} \in \mathbb{Z}_{+}$. By assumption, either $\lambda_{1}$ or $\lambda_{2} \notin \mathbb{Z}_{+} \varpi_{2}$. Therefore at least one of the summands $g\left(\delta_{j}\right)=\left(\mu_{1}, \mu_{2}, \mu_{3}\right)$ is such that $\mu_{1}$ or $\mu_{2}$ resp. does not belong to $\mathbb{Z}_{+} \varpi_{2}$ either. Hence Proposition 6.7 would follow from:

Lemma 6.8. Suppose that $w\left(\delta_{j}\right)=\left(\mu_{1}, \mu_{2}, \mu_{3}\right)$ is such that $\mu_{1} \notin \mathbb{Z}_{+} \varpi_{2}$. Then for each $i=1,2$ the sum $\epsilon_{i}+w\left(\delta_{j}\right)$ belongs to Tens $(G)$.

Proof. The proof of this lemma is a direct computation with the LiE program.

This concludes the proof of Proposition 6.7.

Remark 6.9. Proposition 6.7 implies Corollary 6.2.

Below we observe that certain combinations of the type (14) not covered by Proposition 6.7 nevertheless belong to $\operatorname{Tens}(G)$ :

Lemma 6.10. The following combinations of the type (14) belong to Tens $(G)$ :

$$
\begin{gathered}
\epsilon_{1}+\delta_{5}, \quad \epsilon_{1}+\delta_{9}, \\
\epsilon_{2}+\delta_{6}, \quad \epsilon_{2}+\delta_{9}, \\
\epsilon_{1}+w\left(\delta_{2}\right)+\delta_{6}, \quad \epsilon_{1}+\delta_{4}+\delta_{6}, \quad \epsilon_{1}+u\left(\delta_{2}\right)+\delta_{6}, \\
\epsilon_{2}+\delta_{2}+\delta_{5}, \quad \epsilon_{2}+\delta_{4}+\delta_{5} .
\end{gathered}
$$

Proof. Observe that

$$
\begin{gathered}
\epsilon_{1}+\delta_{5}=2 \delta_{9}, \quad \epsilon_{1}+\delta_{9}=\delta_{2}+\delta_{5}, \\
\epsilon_{2}+\delta_{6}=\delta_{9}+u\left(\delta_{2}\right), \quad \epsilon_{2}+\delta_{9}=\delta_{4}+\delta_{5}
\end{gathered}
$$


and hence they are in Tens $(G)$. The combination $\epsilon_{1}+u\left(\delta_{2}\right)+\delta_{6}$ belongs to $(2 L)^{3}$ and therefore it is in $\operatorname{Tens}(G)$. Moreover

$$
\epsilon_{1}+w\left(\delta_{2}\right)+\delta_{6}=\epsilon_{2}+\delta_{2}+\delta_{5}
$$

Thus it remains to check 3 last combinations in Lemma, which is done by a direct computation with LiE.

By combining Proposition 6.7 with the above lemma we see that it remains to analyze combinations of the following types:

1. $\epsilon_{1}+x \delta_{2}+y w\left(\delta_{2}\right)+z \delta_{4}$.

2. $\epsilon_{2}+x \delta_{2}+y w\left(\delta_{2}\right)+z \delta_{4}$.

3. $\epsilon_{1}+n \delta_{2}+m \delta_{6}$.

4. $\epsilon_{2}+n w\left(\delta_{2}\right)+m \delta_{5}$.

We note that the first two types belongs to $\mathcal{E}_{1}$, the third type belongs to $\mathcal{E}_{2}$ and the last type belongs to $\mathcal{E}_{3}$. Hence we have proved that if $\sigma \in \mathcal{P}(G) \cap L^{3}$ does not belong to $S_{3} \cdot\left(\mathcal{E}_{1} \cup \mathcal{E}_{2} \cup \mathcal{E}_{3}\right)$ then $\sigma \in \operatorname{Tens}(G)$. It is left to show that $\left(\mathcal{E}_{1} \cup \mathcal{E}_{2} \cup \mathcal{E}_{3}\right) \cap T e n s(G)=\emptyset$. This is done in the following three propositions.

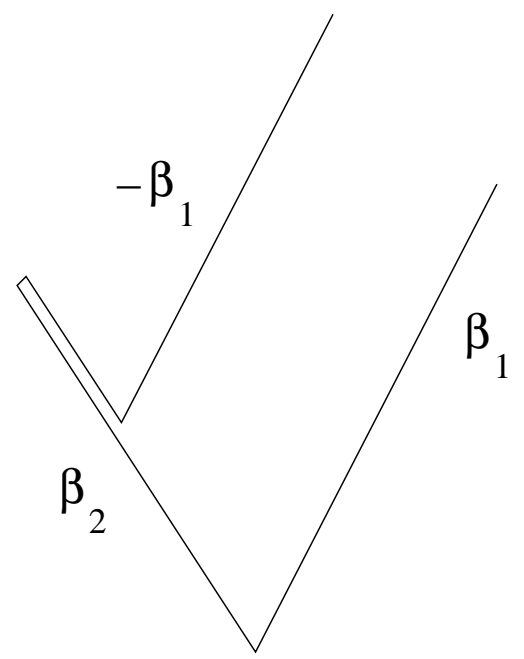

FIG. 11.

Proposition 6.11. For any $n, m \in \mathbb{Z}_{+}$, the triple

$$
\sigma=(\lambda, \nu, \mu)=\epsilon_{1}+n \delta_{2}+m \delta_{6}=\left(\left[\begin{array}{c}
0 \\
1+n+m
\end{array}\right],\left[\begin{array}{c}
0 \\
1+n+2 m
\end{array}\right],\left[\begin{array}{c}
1+3 m \\
0
\end{array}\right]\right)
$$

does not belong to Tens $(G)$. Thus $\mathcal{E}_{1} \cap \operatorname{Tens}(G)=\emptyset$.

Proof. Note that $\lambda, \nu \in \mathbb{N}_{2}, \mu \in \mathbb{N}_{1}$. If $\sigma \in \operatorname{Tens}(G)$ then there exists a Hecke path $p$ connecting $\lambda$ to $\nu$ which is entirely contained in $\Delta$, so that $\operatorname{length}_{\Delta}(p)=$ $(1+3 m) \varpi_{1}$. Let $p$ be modeled on a chain $\eta_{0} \geq \eta_{1} \geq \ldots \geq \eta_{m}$ whose elements are in $W\left(\varpi_{1}\right)$. Note that since $p(0), p(1)$ are on the wall $H_{2}=\mathbb{R} \cdot \varpi_{2}$, and the image of $p$ is contained in $\Delta$, it follows that

$$
\eta_{0} \neq-\beta_{0},-\beta_{2}, \beta_{1}
$$


and

$$
p^{\prime}(1)=\eta_{m} \neq \beta_{2}, \beta_{0} .
$$

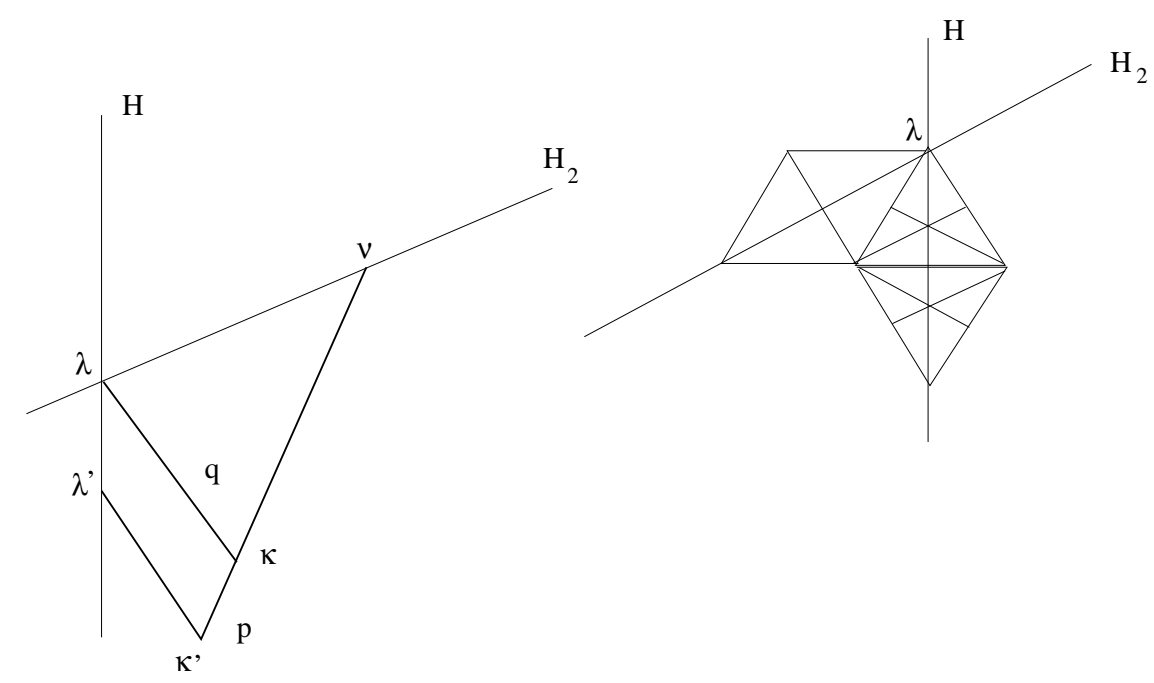

FIG. 12 .

Therefore, the chain $\eta_{0} \geq \eta_{1} \geq \ldots \geq \eta_{m}$ is a subchain of

$$
-\beta_{1} \geq-\beta_{2} \geq \beta_{2} \geq \beta_{1}
$$

and the path $p$ has the shape as in Figure 11. It is clear that $\eta_{m}=\beta_{1}$, for otherwise the path $p$ cannot connect $\lambda$ to $\nu$.

We define a canonical path $q$ which is an $L S(R)$-path connecting $\lambda$ to $\nu$ and which corresponds to the chain $\beta_{2} \geq \beta_{1}$. Then length $\Delta(p)=3 m \varpi_{1}$. Let $\kappa$ denote the break-point of $q$, see Figure 12 .

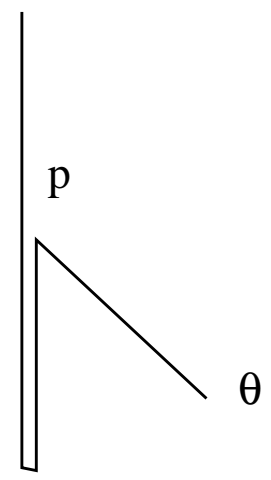

FIG. 13.

If $p$ does not contain the subsegment $\overline{\kappa \nu}$, its orthogonal projection to the horizontal wall $H_{1}$ will have length strictly less than the length of the orthogonal projection of $\overline{\lambda \nu}$, and therefore $p$ cannot connect $\lambda$ to $\nu$. Thus $p$ contains the subsegment $\overline{\kappa \nu}$, let 
$\overline{\kappa^{\prime} \nu}$ denote the maximal geodesic subsegment in $p$ containing $\overline{\kappa \nu}$. Then $\kappa^{\prime}$ has to be a special vertex (since $p$ is a Hecke path). Let $H$ denote the vertical wall through $\lambda$. Let $t$ denote the largest point in $[0,1]$ such that $\lambda^{\prime}=p(t)$ belongs to $H$. Then

$$
\operatorname{length}_{\Delta}(p \mid[t, 1])=\operatorname{length}_{\Delta}(q)=3 m \varpi_{1} .
$$

Suppose that $\kappa^{\prime} \neq \kappa$. Since $\kappa^{\prime}$ is a special vertex, the distance between $\lambda^{\prime}$ and $\lambda$ is at least the length of $\varpi_{2}$, i.e. strictly greater than the length of $\varpi_{1}$. Thus $\kappa^{\prime}=\kappa$.

Therefore let $\overline{\lambda^{\prime \prime} \kappa} \subset \overline{\lambda \kappa}$ denote the largest subsegment contained in $p$. However, if $\lambda^{\prime \prime} \neq \lambda$, we again get a contradiction: The path $p$ is strictly to the right of the wall $H$ which is absurd. $\square$

Proposition 6.12. No triple

$$
\sigma:=(\lambda, \nu, \mu)=\left(\left[\begin{array}{l}
0 \\
x
\end{array}\right],\left[\begin{array}{l}
0 \\
y
\end{array}\right],\left[\begin{array}{l}
1 \\
z
\end{array}\right]\right)
$$

belongs to Tens $(G)$. Thus $\mathcal{E}_{2} \cap$ Tens $(G)=\emptyset$.

Proof. We will need the following two lemmas:

Suppose that $\xi \in H_{2}$ is a special vertex and set $\theta:=\xi+\varpi_{1}$.

Lemma 6.13. There are no Hecke paths $p_{2}:[0,1] \rightarrow \Delta$ so that

- $p_{2}(0)=\theta$,

- $p_{2}(1)$ is a special vertex in $H_{2}$.

- $p_{2}$ is modeled on a subchain in $-\alpha_{1} \geq-\alpha_{2} \geq \alpha_{2}$.

Proof. Under the above assumptions the path $p_{2}$ has the shape as in Figure 13. Therefore the image of $p_{2}$ is contained in the vertical strip $S$ of the width $\left|\varpi_{1}\right| / 2$, see Figure 14. However $S \cap H_{2}$ contains no special vertices. $\square$

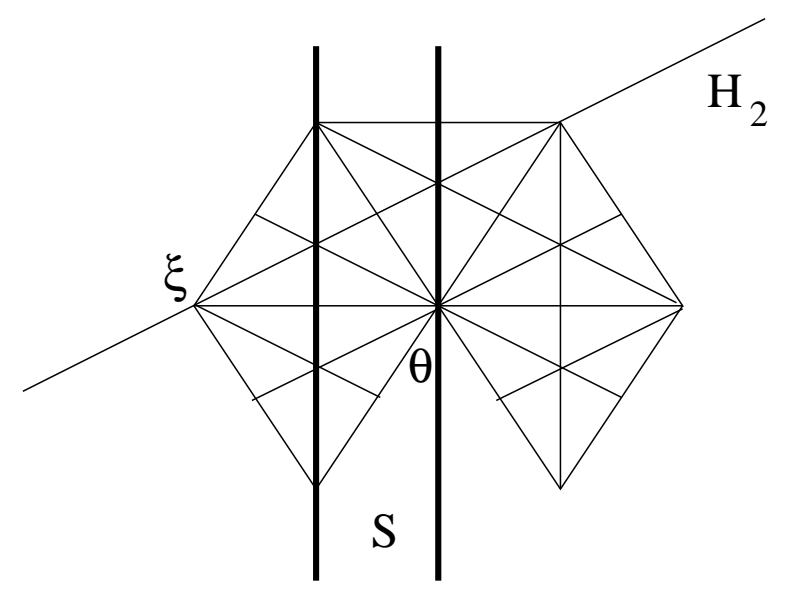

FIG. 14

Set $\theta^{\prime}=\xi+2 \varpi_{1}$

Lemma 6.14. There are no Hecke paths $p_{2}:[0,1] \rightarrow \Delta$ so that - $p_{2}(0)=\theta^{\prime}$, 
- $p_{2}(1)$ is a special vertex in $H_{2}$.

- $p_{2}$ is modeled on the a subchain in $-\alpha_{2} \geq \alpha_{2}$.

Proof. Under the above assumptions the image of $p_{2}$ is contained in the vertical wall $S^{\prime}$ through the point $\theta^{\prime}$, see Figure 15 . However $S \cap H_{2}$ is not a special vertex.

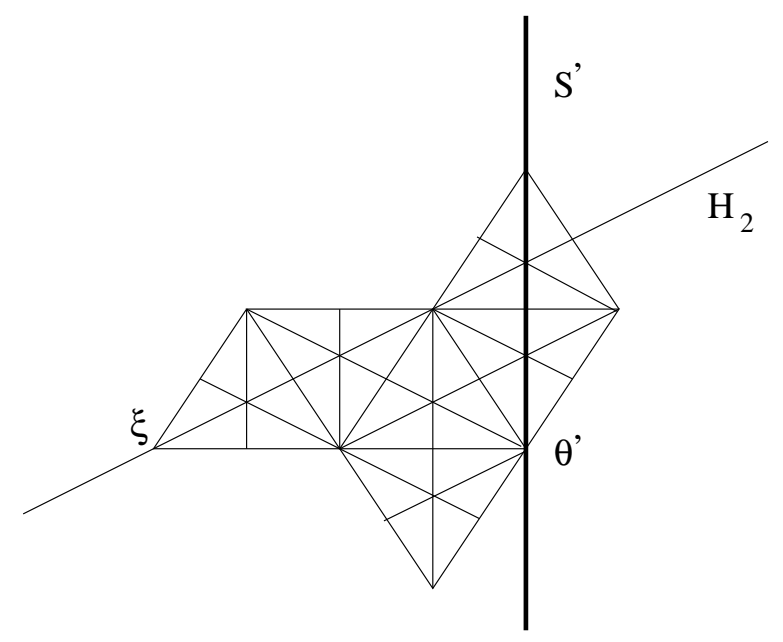

FIG. 15.

Suppose that $p$ is an $L S_{1}(R)$-path in $\Delta$ with

$$
\underline{\text { length }}_{\Delta}(p)=\left(\varpi_{1}, z \varpi_{2}\right)
$$

which is contained in $\Delta$ and connects $\lambda$ to $\nu$. Let $p=p_{1} * p_{2}$ where $p_{i}$ are $L S$ paths.

Since $p_{2}$ is an LS path, if $p_{2}$ has a break-point on the wall $H_{2}$, this point has to be special (see Observation 6.5). Therefore, we can reduce the discussion to the case when $p_{2}$ does not contain nondegenerate subsegments in $H_{2}$, which we assume from now on.

The path $p_{1}$ is either a geodesic path connecting $\lambda$ to $A, B$ or $C$, or $p_{1}(0)=$ $p_{1}(1)=\lambda$ and $p_{1}$ has a unique break-point which is a point of backtracking, see Figure 16.

Lemma 6.15. There is no LS path $p_{1} \subset \Delta$ connecting $\lambda$ to itself such that

$$
\operatorname{length}_{\Delta}\left(p_{1}\right)=\varpi_{1} .
$$

Proof. Since $p_{1}(0)=p_{1}(1) \in H_{2}$, the path $p_{1}$ has to be modeled on a subchain in

$$
-\beta_{1} \geq \beta_{2} \geq \beta_{1} \text {. }
$$

Since length $\Delta\left(p_{1}\right)=\varpi_{1}$, the path $p_{1}$ can have only one break-point, hence it is a backtrack. Thus $p_{1}$ is modeled on a chain of the form $-\eta \geq \eta$, hence the model chain is $-\beta_{1} \geq \beta_{1}$. Such a path is Hecke but not an LS path, see Figure 16.

If $p_{1}(1)=C$ then $p_{1}^{\prime}(1)=\varpi_{1}$. In this case, $p_{2}$ is either modeled on the chain $\alpha_{1} \geq \varpi_{2}$ or $p_{2}$ is geodesic parallel to the wall $H_{2}$. In either case, $p_{2}(1) \neq H_{2}$. 


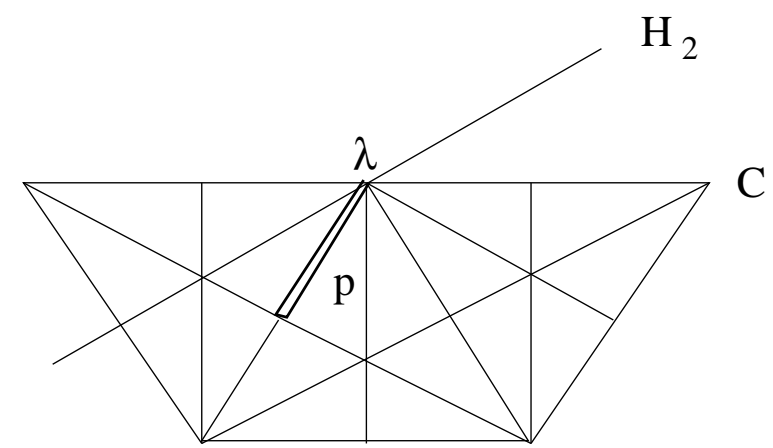

A

FIG. 16.

Thus $p_{2}(0)$ is either $A$ or $B$. Let $p_{2}$ be modeled on a chain $\eta_{0} \geq \eta_{1} \geq \ldots \geq \eta_{m}$ Since $p_{2}(1) \in H$ and $p_{2}^{\prime}(1) \notin \mathbb{R} \varpi_{2}$, it follows that $\eta_{0} \geq \ldots \geq \eta_{m}$ is a subchain in

$$
-\varpi_{2} \geq-\alpha_{1} \geq-\alpha_{2} \geq \alpha_{2}
$$

and the path $p_{2}$ has the shape as in the Figure 17. In particular, the image of $p_{2}$ is contained in the parallel strip $S$ bounded by the vertical walls passing through $p_{2}(0), p_{2}(1)$.
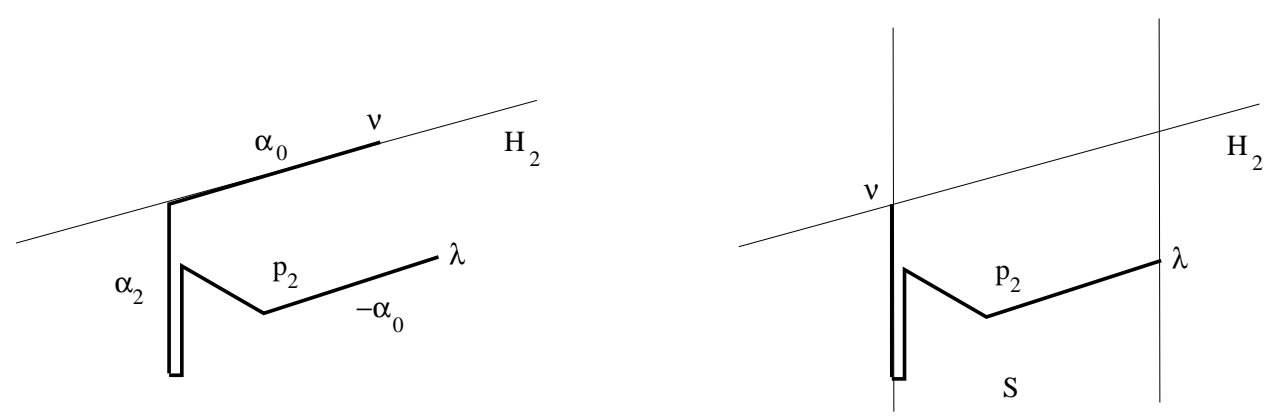

FIG. 17.

Case 1. $p_{2}(0)=B$. The the chain condition satisfied by the concatenation $p_{1} * p_{2}$ at the point $B$ implies that the chain $\eta_{0} \geq \ldots \geq \eta_{m}$ is a subchain in $-\alpha_{2} \geq \alpha_{2}$. This however contradicts Lemma 6.14.

Case 2. $p_{2}(0)=A$. Then the chain $\eta_{0} \geq \ldots \geq \eta_{m}$ is a subchain in $-\alpha_{0} \leq$ $-\alpha_{1} \geq-\alpha_{2} \geq \alpha_{2}$. If it is a subchain in $-\alpha_{1} \geq-\alpha_{2} \geq \alpha_{2}$ we get a contradiction with Lemma 6.14. Therefore the initial maximal geodesic segment $\overline{A D}$ in $p_{2}$ is parallel to the wall $H_{2}$. The assumption that $p_{2}$ is an LS path then implies that the break-point $p_{2}(t)=D$ is a special vertex.

REMARK 6.16. There are Hecke paths $p_{2}:[0,1] \rightarrow \Delta$ for which $p_{2}(0)=A$, $p_{2}(1)$ is a special vertex on $H_{2}$, length ${ }_{\Delta}\left(p_{2}\right) \in \mathbb{N}_{2}$, which are modeled on the chain $-\alpha_{0} \geq \alpha_{2}$. However these paths fail to be LS paths, cf. Observation 6.5. 
Then the restriction $q:=\left.p_{2}\right|_{[t, 1]}$ is a Hecke path in $\Delta$, so that $q(1)=p_{2}(1)$ a special vertex on $H_{2}$ and $q$ is modeled on a subchain in $-\alpha_{1} \geq-\alpha_{2} \geq \alpha_{2}$. We then obtain a contradiction as above.

This concludes the proof of Proposition 6.12.

Proposition 6.17. For any $n, m \in \mathbb{Z}_{+}$, the triple

$$
\sigma=(\mu, \nu, \lambda)=\epsilon_{1}+n w\left(\delta_{2}\right)+m \delta_{5}=\left(\left[\begin{array}{c}
0 \\
1+n+m
\end{array}\right],\left[\begin{array}{c}
0 \\
1+m
\end{array}\right],\left[\begin{array}{c}
1+3 m \\
1+n
\end{array}\right]\right)
$$

does not belong to Tens $(G)$. Thus $\mathcal{E}_{3} \cap \operatorname{Tens}(G)=\emptyset$.

Proof. If $\sigma \in$ Tens $(G)$, then there exits LS path $p \subset \Delta$ connecting $\lambda$ to $\nu$ so that length $_{\Delta}(p)=\mu$. The path $p$ is modeled on a subchain in the chain

$$
-\varpi_{2} \geq-\alpha_{1} \geq-\alpha_{2} \geq \alpha_{2} \geq \omega_{2} .
$$

Therefore the general shape of $p$ is as in Figure 17. Therefore $p$ lies to the right of the vertical wall passing through its last break-point $p(t)$. Similarly to the proof of Proposition 6.12 it suffices to consider the case when $p$ contains no nondegenerate subsegments of the wall $H_{2}$ (see Figure 17), i.e. $p$ is modeled on a subchain in

$$
-\varpi_{2} \geq-\alpha_{1} \geq-\alpha_{2} \geq \alpha_{2} .
$$

Let $H$ denote the vertical wall through $\nu$. Set $\xi:=\nu-\frac{2}{3} \varpi_{2}, \zeta:=\xi+\varpi_{2}$ and let $H^{\prime}$ denote the vertical wall through $\zeta$. Let $\theta \in H$ denote the point so that the triangle $[\xi, \theta, \nu]$ is equilateral. Thus $\nu$ belongs to the interior of the segment $\overline{\xi \zeta}$.

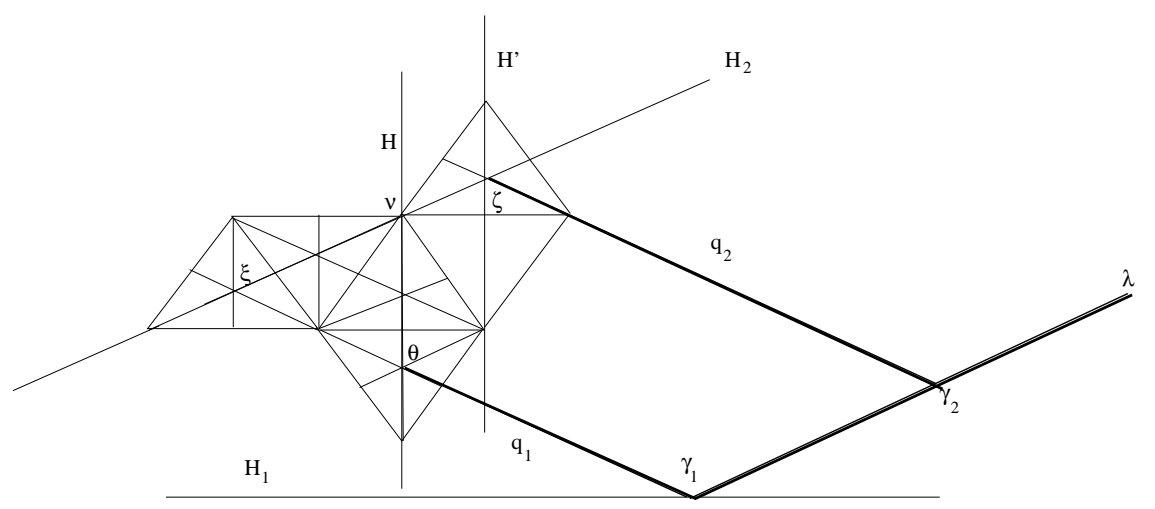

FIG. 18

Define paths $q_{1}, q_{2}$ from $\lambda$ to $\theta$ and $\zeta$ as in Figure 18. These paths have breakpoints at $\gamma_{1}=(1+3 m) \varpi_{1}$ and $\gamma_{2}=(1+3 m) \varpi_{1}+\varpi_{2}$ respectively. Then

$$
\operatorname{length}_{\Delta}\left(q_{1}\right)=\left(n+m+\frac{2}{3}\right) \varpi_{2}, \text { length } \Delta\left(q_{2}\right)=\left(n+m+\frac{2}{3}\right) \varpi_{2} .
$$

Note that $q_{2}$ lies entirely to the right of the wall $H^{\prime}$.

If $p$ does not contain the subsegment $\overline{\lambda \gamma_{2}}$, it also lies to the right of the wall $H^{\prime}$. Such a path cannot connect $\lambda$ to $\nu$. 
Therefore, $p$ contains the segment $\overline{\lambda \gamma_{1}}$. The same argument shows that $p$ cannot have the first break at $\gamma_{2}$. Moreover, unless $p$ is modeled on a chain of the form $-\varpi_{2} \geq-\alpha_{1} \geq \ldots$, it lies to the right of the vertical wall through $\gamma_{1}$. Thus, since $p$ is an LS path, its first break-point has to be a special vertex.

The interior of the segment $\overline{\gamma_{1} \gamma_{2}}$ contains no special vertices, hence the first break of $p$ occurs at $\gamma_{1}$ and $p$ contains a subsegment of $\overline{\gamma_{1} \theta}$.

If $p$ makes a break after $\gamma_{1}$ but before reaching the wall $H$, it lies strictly to the right of $H$ which is again impossible. Thus $p$ contains the subpath $q_{1}$; let $t \in[0,1]$ be such that $p(t)=\theta$. Then

$$
\operatorname{length}_{\Delta}(p \mid[t, 1])=\mu-\operatorname{length}_{\Delta}\left(q_{1}\right)=\frac{1}{3} \varpi_{2} .
$$

However, the distance from $\theta$ to $\nu$ equals $\frac{2}{3}\left|\varpi_{2}\right|>\frac{1}{3}\left|\varpi_{2}\right|$. Thus $p(1) \neq \nu$. Contradiction.

This concludes the proof of Theorem 6.1.

\section{REFERENCES}

[1] W. Ballmann, "Lectures on spaces of nonpositive curvature. With an appendix by Misha Brin." DMV Seminar, vol. 25. Birkhauser Verlag, Basel, 1995.

[2] A. Berenstein and R. SjamaAr, Coadjoint orbits, moment polytopes, and the HilbertMumford criterion, Journ. Amer. Math. Soc., 13:2 (2000), pp. 433-466.

[3] A. Berenstein and A. Zelevinsky, Tensor product multiplicities, canonical bases and totally positive varieties, Invent. Math., 143:1 (2001), pp. 77-128.

[4] N. Bourbaki, Lie groups and Lie algebras, Chap. 4, 5, 6. Springer Verlag, 2002.

[5] K. Brown, Buildings, Springer-Verlag, New York, 1989.

[6] F. Bruhat and J. Tits, Groupes reductifs sur un corps local, Inst. Hautes Études Sci. Publ. Math., 41 (1972), pp. 5-251.

[7] R. Cluckers, Presburger sets and p-minimal fields, J. Symbolic Logic, 68:1 (2003), pp. 153162.

[8] W. Fulton and J. Harris, "Representation theory. A first course," Graduate Texts in Math., vol. 129, Springer Verlag, 1991.

[9] J. E. Humphreys, Introduction to Lie algebras and representation theory, Graduate Texts in Mathematics, 9. Springer-Verlag, New York-Berlin, 1978.

[10] M. Kapovich, B. Leeb and J. J. Millson, Convex functions on symmetric spaces, side lengths of polygons and the stability inequalities for weighted configurations at infinity, Preprint, June 2004, arXiv:math.DG/0311486.

[11] M. Kapovich, B. Leeb and J. J. Millson, Polygons in buildings and their refined side-lengths, Preprint, June 2004, arXiv:math.MG/0406305.

[12] M. Kapovich, B. Leeb and J. J. Millson, Polygons in symmetric spaces and buildings with applications to algebra, Preprint, 2004, arXiv:math.RT/0210256, To appear in Memoirs of AMS.

[13] M. Kapovich And J. J. Millson, A path model for geodesics in Euclidean buildings and its applications to representation theory, Preprint, 2004, arXiv:math.RT/0411182.

[14] B. Kleiner And B. Leeb, Rigidity of quasi-isometries for symmetric spaces and Euclidean buildings, Publ. Math. IHES, 86 (1997), pp. 115-197.

[15] A. Knutson and T. TaO, The honeycomb model of $\mathrm{GL}_{n}(\mathbb{C})$ tensor products. I. Proof of the saturation conjecture, J. Amer. Math. Soc., 12:4 (1999), pp. 1055-1090.

[16] S. Kumar, B. Leeb And J. J. Millson, The generalized triangle inequalities for rank 3 symmetric space of noncompact type, In: "Explorations in complex and Riemannian geometry (Papers dedicated to Robert Greene)," Contemporary Math., 332 (2003), pp. 171-195.

[17] M. C. Laskowski, An application of Kochen's Theorem, J. Symbolic Logic, 68:4 (2003), pp. $1181-1188$.

[18] P. Littelmann, Paths and root operators in representation theory, Annals of Math. (2), 142:3 (1995), pp. 499-525.

[19] M. Ronan, Lectures on buildings, Perspectives in Mathematics, 7. Academic Press, Inc., 1989. 
[20] G. Rousseau, Euclidean buildings, Lectures at Ecole d'ete de Mathematiques "Nonpositively curved geometries, discrete groups and rigidities", Institut Fourier, Grenoble, 2004. 
M. KAPOVICH AND J. J. MILLSON 